\title{
Independent information from PET, CSF and plasma biomarkers of tau pathology in Alzheimer's disease
}

\author{
Rik Ossenkoppele ${ }^{1,2^{*}}$, Juhan Reimand ${ }^{2,3,4}$, Ruben Smith $^{1,5}$, Antoine Leuzy ${ }^{1}$, \\ Olof Strandberg ${ }^{1}$, Sebastian Palmqvist ${ }^{1,6}$, Erik Stomrud ${ }^{1,6}$, Henrik Zetterberg ${ }^{7,8,9,10}$, \\ the Alzheimer's Disease Neuroimaging Initiative\#, Philip Scheltens ${ }^{2}$, Jeffrey L. Dage ${ }^{11}$, \\ Femke Bouwman ${ }^{2}$, Kaj Blennow ${ }^{7,8}$, Niklas Mattsson-Carlgren ${ }^{1,5,12}$ \\ Shorena Janelidze ${ }^{1} \&$ Oskar Hansson ${ }^{1,6^{*}}$
}

\begin{abstract}
${ }^{1}$ Lund University, Clinical Memory Research Unit, Lund, Sweden, ${ }^{2}$ Alzheimer Center Amsterdam, Department of Neurology, Amsterdam Neuroscience, Vrije Universiteit Amsterdam, Amsterdam UMC, Amsterdam, The Netherlands, ${ }^{3}$ Department of Health Technologies, Tallinn University of Technology, Tallinn, Estonia, ${ }^{4}$ Radiology Centre, North Estonia Medical Centre, Tallinn, Estonia, ${ }^{5}$ Department of Neurology, Skåne University Hospital, Lund, Sweden, ${ }^{6}$ Memory Clinic, Skåne University Hospital, Malmö, Sweden, ${ }^{7}$ Clinical Neurochemistry Laboratory, Sahlgrenska University Hospital, Mölndal, Sweden ${ }^{8}$ Department of Psychiatry and Neurochemistry, Institute of Neuroscience and Physiology, The Sahlgrenska Academy at the University of Gothenburg, Mölndal, Sweden, ${ }^{9}$ Department of Neurodegenerative Disease, UCL Institute of Neurology, London, UK, ${ }^{10}$ UK Dementia Research Institute at UCL, London, UK, ${ }^{11}$ Eli Lilly and Company, Indianapolis, USA, ${ }^{12}$ Wallenberg Centre for Molecular Medicine, Lund University, Lund, Sweden.
\end{abstract}

\# Data used in preparation of this article were obtained from the Alzheimer's Disease Neuroimaging Initiative (ADNI) database (adni.loni.usc.edu). As such, the investigators within the ADNI contributed to the design and implementation of ADNI and/or provided data but did not participate in analysis or writing of this report. A complete listing of ADNI investigators can be found at: http://adni.loni.usc.edu/wp-content/uploads/how to apply/ADNI_Acknowledgement List.pdf

\section{Corresponding authors:}

* Rik Ossenkoppele (r.ossenkoppele@amsterdamumc.nl), Lund University, Clinical Memory Research Unit, Malmö, Sweden \& VU University Medical Center, Department of Neurology and Alzheimer Center, Amsterdam Neuroscience, Amsterdam, the Netherlands, \& Oskar Hansson (oskar.hansson@med.lu.se), Lund University, Clinical Memory Research Unit, Malmö, Sweden \& Memory Clinic, Skåne University Hospital, Malmö, Sweden. 


\begin{abstract}
PET, CSF and plasma biomarkers of tau pathology may be differentially associated with Alzheimer's disease $(\mathrm{AD})$ related demographic, cognitive, genetic and neuroimaging markers. We examined 771 participants with normal cognition, mild cognitive impairment or dementia from BioFINDER-2 $(n=400)$ and ADNI $(n=371)$. All had tau-PET $\left(\left[{ }^{18} \mathrm{~F}\right] \mathrm{RO} 948\right.$ in BioFINDER-2, $\left[{ }^{18} \mathrm{~F}\right]$ flortaucipir in ADNI) and CSF p-tau181 biomarkers available. Plasma ptau181 and plasma/CSF p-tau217 were available in BioFINDER-2 only. Concordance between PET, CSF and plasma tau biomarkers ranged between $66 \%$ and $95 \%$. Across the whole group, ridge regression models showed that increased CSF and plasma p-tau181 and p-tau217 levels were independently of tau PET associated with higher age, and APOE\&4-carriership and A $\beta$ positivity, while increased tau-PET signal in the temporal cortex was associated with worse cognitive performance and reduced cortical thickness. We conclude that biofluid and neuroimaging markers of tau pathology convey partly independent information, with CSF and plasma p-tau181 and p-tau217 levels being more tightly linked with early markers of AD (especially $\mathrm{A} \beta$ pathology), while tau-PET shows the strongest associations with cognitive and neurodegenerative markers of disease progression.
\end{abstract}




\section{INTRODUCTION}

The core neuropathological features of Alzheimer's disease (AD) are amyloid- $\beta(A \beta)$ plaques and hyperphosphorylated tau (p-tau) in neuronal neurofibrillary tangles and neuropil threads. ${ }^{1}$ While $\mathrm{A} \beta$ pathology is often considered a key initiator of $\mathrm{AD}$ development (potentially through facilitating the spread of tau pathology ${ }^{2}$ ), the phosphorylation, release and aggregation of tau proteins is tightly linked to the clinical and biological progression of AD. ${ }^{3-7}$ Major scientific breakthroughs over the past decades now enable the detection of tau pathology in cerebrospinal fluid (CSF), using positron emission tomography (PET) and, most recently, in blood. ${ }^{8-11}$

Although these three biomarker modalities are reflecting tau pathology, there are important differences between them. For example, in CSF and plasma specific soluble variants of tau (e.g., p-tau181 or p-tau217) are measured, ${ }^{12-18}$ while tau PET ligands bind aggregated nonsoluble paired helical filaments of tau. ${ }^{19-23}$ PET and fluid biomarkers thus measure different aspects of abnormalities in tau metabolism. Moreover, previous studies have shown that CSF p-tau markers become abnormal prior to tau PET and may thus be more sensitive biomarkers for early AD. ${ }^{24-26}$ Similarly, there is emerging evidence that alterations in plasma p-tau levels also occur early in the disease process. ${ }^{4,17,27-31}$ Despite the aforementioned differences among biofluid- and PET-based tau biomarkers, they are often considered interchangeable. For example, CSF p-tau and tau PET are both incorporated as markers of tau pathology in the most recent research criteria for $\mathrm{AD}^{32}$, and both can be used to define " $\mathrm{T}$ " (tau) status in the $\mathrm{AT}(\mathrm{N})$ classification system. ${ }^{33}$

In this study, we investigated whether PET, CSF and plasma biomarkers of tau pathology are differentially associated with AD-related demographic, cognitive, genetic and neuroimaging markers. We hypothesized that the three modalities would show significant differences in 
associations with distinct $\mathrm{AD}$ features, and thus partially convey independent information. Exploring this hypothesis might provide insight into the clinical and neurobiological factors related to discrepant results between PET-, CSF- and blood-based biomarkers of tau pathology. Additionally, in light of the recent FDA approval of one of the tau PET tracers (i.e., $\left[{ }^{18} \mathrm{~F}\right]$ flortaucipir $\left.{ }^{34}\right)$, and the rapid development of blood-based p-tau biomarkers, clinicians in specialized care settings may soon have multiple tau biomarker options at their disposal. It is therefore of high clinical relevance to determine the degree of agreement between the tau biomarkers and to identify potential scenarios where one tau biomarker might be preferred over the other(s).

\section{RESULTS}

\section{Study participants}

For this study, we stratified participants by their cognitive status into cognitively unimpaired (CU) and symptomatic groups (combining MCI and dementia, Table 1). As expected in both BioFINDER-2 and ADNI, participants in the symptomatic group were older, had more often pathological levels of both tau and $\mathrm{A} \beta$ biomarkers, and demonstrated worse cognitive performance and greater atrophy on MRI compared to CU individuals.

\section{Associations between tau biomarkers and AD-related features}

First, we examined the correlations between continuous tau biomarkers and other AD-related features. Overall, there was a moderate to strong positive correlation between PET, CSF and plasma tau biomarkers (range: $0.46-0.98$, all $\mathrm{p}<0.001$, Figure 1a). In addition, tau biomarkers were positively correlated with age (range: 0.22 for plasma p-tau217 to 0.35 for plasma $\mathrm{p}$ tau181, all $\mathrm{p}<0.001$ ) and amyloid PET global SUVR (range: 0.48 for plasma p-tau181 to 0.78 for PET entorhinal cortex, all $\mathrm{p}<0.001)$. Furthermore, tau biomarkers were negatively 
correlated with CSF A $\beta_{42 / 40}$ ratio (range: -0.45 for plasma p-tau181 to -0.66 for CSF p-tau181, all $\mathrm{p}<0.001$ ), cognitive tests scores (e.g., MMSE, range: -0.36 for plasma $p$-tau181 to -0.75 for PET temporal meta-ROI, all $\mathrm{p}<0.001$ ) and MRI measures (e.g., AD-signature cortical thickness, range: -0.39 for plasma $\mathrm{p}$-tau181 to -0.62 for PET entorhinal cortex, all $\mathrm{p}<0.001$ Figure 1b). In general, the correlation coefficients for AD-related features were slightly lower for plasma p-tau181. Furthermore, p-tau217 in both plasma and CSF showed a higher correlation with tau PET than p-tau181 in plasma or CSF. Similar associations were observed for the available variables in ADNI (Figure 1c).

Concordance of PET, CSF and plasma tau biomarkers

Next, we examined the concordance between PET, CSF and plasma tau biomarkers in the BioFINDER-2 cohort. The concordance between tau PET standardized uptake value ratios (SUVR) in the temporal meta-ROI and CSF p-tau181 was $83 \%$ (Figure 2). Of the 17\% discordant participants, $16 \%$ showed isolated tau positivity on CSF p-tau181 (CSF+), while only $1 \%$ were positive for tau PET $(\mathrm{PET}+)$ and negative for CSF $\mathrm{p}$-tau181. Tau PET was concordant with plasma p-tau181 in $80 \%$ (5\% plasma+/PET-, 15\% PET+/plasma-), with plasma p-tau 217 in $86 \%$ (12\% plasma+/PET-, $2 \%$ PET+/plasma-), and with CSF p-tau 217 in $80 \%$ of cases $(19 \% \mathrm{CSF}+\mathrm{PET}-, 1 \% \mathrm{PET}+/ \mathrm{CSF}$, Figure 2$)$.

The concordance between fluid tau biomarkers ranged between $66 \%$ (CSF p-tau217 vs plasma p-tau181), 70\% (CSF p-tau181 vs plasma p-tau181), 73\% (plasma p-tau181 vs plasma ptau217), 82\% (CSF p-tau181 vs plasma p-tau217), 83\% (CSF p-tau217 vs plasma p-tau217) and 95\% (CSF p-tau181 vs CSF p-tau217, Supplementary figure 1). Tau PET SUVR in the entorhinal cortex showed a concordance of $87 \%$ with CSF p-tau217 (12\% CSF+/PET-, 1\% PET $+/$ CSF-) and of $84 \%$ with plasma p-tau217 (9\% plasma+/PET-, 6\% PET+/plasma-), 
Supplementary figure 1). Overall, in terms of biomarker discordance, a biofluid+/PETprofile was more common than a biofluid-/PET+ profile, with the exception of plasma $\mathrm{p}$ $\operatorname{tau} 181$.

Partly differential associations between tau PET and CSF p-tau181 vs AD-related features

Based on three sets of ridge regression models (model 1: CSF p-tau181, model 2: tau PET SUVR in the temporal meta-ROI, model 3: CSF and PET combined) for each AD-related feature, we examined the possible differential associations for either tau PET or CSF p-tau181 to other AD-related features (Figure 3a). Analyses across the whole group indicated that CSF p-tau181 was independently from tau PET associated to risk factors for AD (i.e., age and $A P O E$ $\varepsilon 4$ carriership) and $A \beta$ pathology, whereas tau PET was more strongly associated with indicators of disease progression including lower cognitive test scores and reduced cortical thickness. When stratifying for cognitive status (i.e., CU vs MCI/dementia), this pattern was similar for CSF in CU and for PET in the symptomatic stages. Additionally, CSF was more strongly associated with MMSE and executive functioning composite score in CU individuals. These patterns of results were consistent when investigating percentual change of $\beta$ coefficients (Supplementary table 2) and when comparing the R-squared values between the simple models for PET and CSF (Supplementary table 3). Replication of the analyses in ADNI revealed overall similar results, except that the stronger association of CSF with cognitive measures in CU individuals observed in BioFINDER-2 was not present in ADNI (Figure 3b).

Relative importance of AD features in predicting CSF p-tau181 and tau PET levels Next, we explored the association between CSF p-tau181 and tau PET SUVR in a temporal meta-ROI with AD-related features using regression tree models in both BioFINDER-2 
(Figure 4a) and in ADNI (Figure 4b). The advantage of regression tree models is that all variables are modelled together, resulting in an estimation of how important each AD-related feature is relative to the other features for PET and CSF separately. We found that amyloid PET retention and the CSF $A \beta_{42 / 40}$ ratio were among the most important predictors of tau biomarker levels for most models, but they showed tau biomarker modality specific effects. For example, the CSF $A \beta_{42 / 40}$ ratio was an important predictor of CSF p-tau181 levels in both CU (median VIM [95\% confidence intervals]; BioFINDER-2: 1.23 [1.18-1.32], rank \#2; ADNI: 1.35 [1.25-1.51], rank \#1) and MCI/dementia (BioFINDER-2: 1.43 [1.32-1.59], rank \#1; ADNI: 1.21 [1.10, 1.39], rank \#1) groups, while it was a more important predictor of tau PET in the CU group (BioFINDER-2: 0.67 [0.57-0.80], rank \#3; ADNI: 0.91 [0.75-1.05], rank \#1), but to a lesser extent in MCI/dementia (BioFINDER-2: 0.86 [0.72-1.06], rank \#9; ADNI: 0.75 [0.67-0.83], rank \#4). In CU, age was an important predictor for both CSF p-tau181 and tau PET (ranks varying from \#2 to \#4), whereas it was not an important predictor for either tau biomarker in the symptomatic stages (ranks varying from \#6 to \#9). In the symptomatic stages, memory z-score was the most informative out of cognitive tests for all models, which was more pronounced for tau PET (ranked \#2 in both cohorts) than for CSF p-tau181 (ranked \#4 in both cohorts). These results are largely congruent with the information obtained using the ridge regression models.

\section{Exploring plasma p-tau markers, p-tau217 epitopes and entorhinal cortex tau PET}

Finally, we used the aforementioned ridge regression models to explore associations of the AD-related features with plasma p-tau181, plasma p-tau217 and CSF p-tau217 (all compared to temporal meta-ROI tau PET) and with entorhinal cortex tau PET SUVR (in comparison to CSF p-tau181). When comparing plasma p-tau181 and tau PET in the temporal meta-ROI, plasma p-tau181 only showed a stronger association with age, while tau PET most strongly 
associated with all cognitive test scores and MRI measures (Figure 5a). Compared to tau PET, plasma p-tau217 was more strongly associated with $A P O E$ \&4 carriership, amyloid PET and the CSF A $\beta_{42 / 40}$ ratio, while tau PET was more strongly associated with cognitive decline and MRI-based atrophy compared to plasma p-tau217 (Figure 5b). Replacing CSF p-tau181 with CSF p-tau217 yielded essentially the same results as in our main analysis (Figure 5c), although it should be noted that the R-squared values from the simple models were slightly but consistently higher for CSF p-tau217 compared to CSF p-tau181 (Supplementary table 4), with the exception of age. We additionally compared CSF p-tau217 to tau PET in the entorhinal cortex (Supplementary figure 2). Contrary to analyses including the temporal meta-ROI, CSF p-tau217 was no longer independently associated with age and $A P O E \varepsilon 4$ carriership, and the stronger associations between tau PET and cognitive decline in the symptomatic stage were no longer found.

\section{DISCUSSION}

In this study, we aimed to investigate whether PET, CSF and plasma biomarkers of tau pathology in $\mathrm{AD}$ are comparable to each other or carry unique information about $\mathrm{AD}$-related demographic, cognitive, genetic and neuroimaging markers. First, we showed that the concordance ranged between 66\% (CSF p-tau217 vs plasma p-tau181) and 95\% (CSF p-tau181 vs CSF p-tau217) across all tau biomarkers, and between 80\% (tau PET vs plasma ptau181/CSF p-tau217) and 86\% (tau PET vs plasma p-tau217) for biofluid- vs neuroimagingbased tau biomarkers. Ridge regression models showed that increased CSF and plasma ptau181 and p-tau217 levels were independently associated with aging, and $A P O E \varepsilon 4$ and $\mathrm{A} \beta$ positivity, while increased temporal meta-ROI tau PET retention was more strongly associated with worse cognitive performance and reduced cortical thickness. The majority of results were consistent between the discovery cohort (i.e., BioFINDER-2) and the replication cohort (i.e., 
ADNI). The data suggest that biofluid tau biomarkers are more tightly linked with early markers of AD (especially A $\beta$ pathology), while tau PET showed strongest associations with cognitive and neurodegenerative markers of disease progression. Overall, the results support our hypothesis that the three tau biomarker modalities provide partially independent information.

The concordance between tau PET vs p-tau181 and p-tau217 in CSF and in plasma ranged from $80 \%$ to $86 \%$. An optimistic interpretation of this level of agreement would be that CSF and especially plasma biomarkers offer cheaper and more scalable alternatives compared to tau PET when the objective is to obtain evidence of the presence or absence of pathological levels of hyperphosphorylated tau. On the other hand, there is also a substantial mismatch of up to $20 \%$ between the neuroimaging vs biofluid markers that can have important ramifications for their application in clinical, investigational and clinical trial settings. One can draw a parallel with studies comparing amyloid PET vs CSF A $\beta$ levels that consistently observed discordance rates of $\sim 10-20 \% .{ }^{35-38}$ This biomarker discordance was demonstrated to be impactful. For example, persons with abnormal CSF A $\beta$ levels but a normal amyloid PET scan were more likely to accumulate more $\mathrm{A} \beta$ pathology over time and to show faster clinical progression than persons with normal CSF A $\beta$ levels but an abnormal amyloid PET. ${ }^{26,35,39,40}$ Moreover, studies have shown that CSF $A \beta_{42}$ may yield false positive results in certain neurological conditions evoking a neuroinflammatory response $\mathrm{e}^{41-43}$, although this can partially be accounted for by using an $A \beta_{42 / 40}$ or $A \beta_{42} / p$-tau ratio. The intended use of biomarkers is thus highly context dependent, and multiple factors like patient characteristics, goal of the biomarker assessment, and availability of resources and expertise may weigh in. Although the results of this study are not conclusive, they are in line with previous work highlighting that tau PET might be most useful for the differential diagnosis of dementia (thus late-stage disease) and for tracking 
disease progression ${ }^{44-49}$, while CSF and plasma tau biomarkers are more sensitive markers that can used to detect $\mathrm{AD}$ in its earliest stages. ${ }^{25,27-29,31}$ Altogether, these findings challenge the notion that the different tau biomarkers can be used interchangeably. ${ }^{50}$

The independent information provided by PET, CSF and plasma biomarkers may be explained by some inherent biological differences. Autopsy studies have shown that tau PET (at least with the tracer $\left[{ }^{18} \mathrm{~F}\right]$ flortaucipir) is not very sensitive and generally reveals neurofibrillary tangle pathology in Braak stage 4 or higher. ${ }^{34,51}$ On the other hand, CSF and plasma p-tau mirror concurrent abnormalities in tau metabolism such as increased phosphorylation and release of soluble tau from damaged neurons. ${ }^{4,52}$ Soluble p-tau levels in CSF and plasma are thus state markers reflecting the balance between production and clearance of tau at time of lumbar puncture or blood draw, while the insoluble tau aggregates measured with PET are likely the product of processes that have occurred over the entire disease duration, making tau PET a stage marker. The detection of tau pathology in a relatively late stage and in a more mature (insoluble) conformation with PET is in line with its strong cognitive and neurodegenerative correlates (i.e., markers of disease progression), while the detection of early abnormalities in tau metabolism with CSF and plasma is consistent with their strong associations with early indicators of $\mathrm{AD}$ like aging and $A P O E \varepsilon 4$ and $\mathrm{A} \beta$ positivity. In addition to differences between modalities, there were also within-modality differences when using ptau181 or p-tau217. In line with previous studies ${ }^{13,17}$, p-tau217 showed subtly stronger associations with tau PET measures and other AD-features than p-tau181, which could in turn be explained by stronger relationships with the quantity of neurofibrillary tangle pathology and neuropil threads for antibodies recognizing p-tau217 vs p-tau181. ${ }^{53}$

\section{Strengths and limitations}


Strengths of the study include the large and comprehensive dataset and the inclusion of a replication cohort. There are also some limitations. First, plasma p-tau181 and p-tau217 were not available in ADNI at the time of tau PET (that was performed six years later ${ }^{30}$ ) and CSF ptau217 was not determined. Therefore, we could only replicate the tau PET vs CSF p-tau181 analysis in ADNI. Moreover, we used different tau PET tracers and CSF assays between BioFINDER and ADNI. Second, the sample size of symptomatic patients was insufficient to stratify by MCI and AD dementia and these groups were therefore pooled. Future studies with larger sample sizes should test whether results differ between MCI and AD dementia.

\section{Future directions}

In addition to investigational and research settings, biomarkers have become an integrated part of clinical trials. For example, CSF p-tau and tau PET have served as a secondary outcome measures in clinical trials testing the efficacy of disease modifying treatments targeting the $\mathrm{A} \beta$ pathway. ${ }^{54-56}$ Until recently, (pre-)screening and/or selection of clinical trial participants was only done using A $\beta$ PET and/or CSF biomarkers. ${ }^{57}$ A recent successful phase II clinical trial with the $A \beta$ antibody donanemab, however, took an innovative approach by selecting $A \beta$ positive individuals $\mathrm{MCI} / \mathrm{mild}$ dementia with intermediate levels of tau pathology based on a PET scan. ${ }^{58}$ Furthermore, an underpowered exploratory analysis suggested that clinical benefit may be associated with the lower tau PET SUVR range. This clinical trial served as the first example for tau PET biomarkers as a selection tool for trial participants. Future work is needed to establish whether cheaper and more scalable plasma and/or CSF tau biomarkers are suitable alternatives to tau PET. A key question is whether biofluid markers show a greater dynamic range at earlier pathological stages, while PET continues to increase in the advanced disease stages (i.e., plateaus at a later stage of the disease). As clinical trial design continues to evolve, it is important to consider the use of biomarkers in those trials and how those markers may or 
may not be useful in primary care settings to aid in management of patients through helping inform on when to start or stop treatment.

\section{MATERIALS AND METHODS}

\section{Participants}

We included a total of 771 participants from the Swedish BioFINDER-2 study at Lund University (discovery cohort, $\mathrm{n}=400$ ) and the Alzheimer's disease neuroimaging initiative (ADNI, replication cohort, $\mathrm{n}=371$ ), including 461 cognitively unimpaired (CU) individuals (BioFINDER-2: 219, ADNI: 242) and 310 individuals diagnosed with mild cognitive impairment (MCI) or AD dementia according to NIA-AA diagnostic criteria ${ }^{59,60}$ (BioFINDER2: 181, ADNI: 129). All participants had tau PET and CSF data available, while plasma p-tau biomarkers (at time of tau PET and lumbar puncture) were only available for the Swedish BioFINDER-2 participants. In addition, all participants underwent a medical history and neurological examination, MRI, $A P O E$ genotyping and a neuropsychological test battery that included the Mini-Mental State Examination (MMSE) and domain specific tests for memory, executive functioning, language and visuospatial abilities. ${ }^{61-63}$ Written informed consent was obtained from all participants and local institutional review boards for human research approved the study. The study was performed in accordance with the ethical standards as laid down in the 1964 Declaration of Helsinki and its later amendments.

\section{MRI data}

In BioFINDER-2, a high- resolution T1-weighted MRI was acquired (3T MAGNETOM Prisma; Siemens Healthineers), while multiple 1.5T and 3T MRI scanners were used in the multi-center ADNI study. ${ }^{64}$ MRI data were processed using previously reported procedures. $^{44,45,63,65,66}$ Briefly, cortical reconstruction and volumetric segmentation were 
performed with the FreeSurfer (v6.0) image analysis pipelines (http://surfer.nmr.mgh.harvard.edu/). The MP-RAGE images underwent correction for intensity homogeneity ${ }^{67}$, removal of non-brain tissue ${ }^{68}$, and segmentation into grey matter (GM) and white matter (WM) with intensity gradient and connectivity among voxels ${ }^{69}$. Cortical thickness was measured as the distance from the GM/WM boundary to the corresponding pial surface $^{70}$. Reconstructed data sets were visually inspected for accuracy, and segmentation errors were corrected. We computed hippocampal volumes (adjusted for total intracranial volume) and $\mathrm{AD}$-signature cortical thickness comprising bilateral entorhinal, inferior and middle temporal and fusiform cortex. ${ }^{71}$

\section{Amyloid PET and CSF}

In the BioFINDER-2 study, amyloid PET was performed using $\left[{ }^{18} \mathrm{~F}\right]$ flutemetamol on a digital Discovery MI scanner (GE Healthcare). SUVR images were created for the 90 to 110 minutes post-injection interval using the pons as reference region. In ADNI, amyloid PET was performed using $\left[{ }^{18} \mathrm{~F}\right]$ florbetapir $(\mathrm{n}=221,50-70$ minutes post-injection, whole cerebellum reference region) or $\left[{ }^{18} \mathrm{~F}\right]$ florbetaben $(\mathrm{n}=150,90-110$, minutes post-injection, whole cerebellum reference region) on multiple PET scanners. SUVR values were re-scaled onto the Centiloid scale (for $\left[{ }^{18} \mathrm{~F}\right]$ florbetapir: [196.9* SUVR $]-196.03$, for $\left[{ }^{18} \mathrm{~F}\right]$ florbetaben: [159.08 * SUVR $]-$ $151.65)^{72}$ to enable pooled analysis. For BioFINDER-2 and ADNI, the CSF A $\beta_{42 / 40}$ ratio was determined using the MSD platform (Meso Scale Discovery) and Elecsys immunoassays (Roche Diagnostics, Basel), respectively.

\section{Tau PET}

PET images were processed using previously reported procedures. ${ }^{45,65,66,73}$ In the BioFINDER2 study, tau PET was performed using $\left[{ }^{18} \mathrm{~F}\right] \mathrm{RO} 948$ on a digital Discovery MI scanner (GE 
Healthcare). Standardized uptake value ratio (SUVR) images were created for the 70 to 90 minutes post-injection interval using the inferior cerebellar cortex as the reference region. In ADNI, tau PET was performed using $\left[{ }^{18} \mathrm{~F}\right]$ flortaucipir and SUVR images were created for the 80-100 post-injection interval using inferior cerebellar cortex as the reference region using a previously published approach. ${ }^{73}$ In line with previous work from our group and others ${ }^{44,45,65,74,75}$, we used a temporal meta- $\mathrm{ROI}^{71}$ comprising a weighted average of entorhinal, amygdala, parahippocampal, fusiform and inferior and middle temporal ROIs for the primary analysis. For the concordance analysis with CSF and plasma p-tau biomarkers, we binarized temporal meta-ROI tau PET retention using previously established cut-offs of 1.36 $\left(\left[{ }^{18} \mathrm{~F}\right] \mathrm{RO} 948\right)$ and 1.34 ([ $\left.{ }^{18} \mathrm{~F}\right]$ flortaucipir $)$ SUVR based on mean $+(2 \mathrm{x}$ standard deviation $)$ uptake in elderly cognitively normal individuals and mean $+(2 \mathrm{x}$ standard deviation $)$ uptake in young cognitively normal individuals, respectively. ${ }^{65}$ Because previous studies suggested that CSF p-tau and plasma p-tau may become abnormal prior to tau PET, we performed a sensitivity analysis using entorhinal cortex $\operatorname{SUVR}^{76,77}$ (a brain region affected early in $\mathrm{AD}^{78}$ ), using previously established cut-offs of $1.48\left(\left[{ }^{18} \mathrm{~F}\right] \mathrm{RO} 948\right)$ and 1.39 ([ $\left.{ }^{18} \mathrm{~F}\right]$ flortaucipir) SUVR. ${ }^{65}$

\section{CSF p-tau biomarkers}

CSF samples were derived from lumbar puncture performed within 12 months from the tau PET scan. The procedures and analysis of CSF followed the Alzheimer's Association Flow Chart for CSF biomarkers ${ }^{79}$ and were performed by technicians blinded to the clinical and imaging data. In BioFINDER-2, analysis of CSF p-tau181 and p-tau217 was performed at Eli Lilly and Company using the MSD platform. ${ }^{13}$ In ADNI, CSF p-tau181 was quantified using Elecsys immunoassays (Roche, Basel, Switzerland), while CSF p-tau217 was not available. ${ }^{80}$ For concordance analyses (in BioFINDER-2 only), we used a previously established cut-off for CSF p-tau2 17 of $101.95 \mathrm{pg} / \mathrm{mL}$, based on the mean $+(2 \mathrm{x}$ standard deviation $)$ in a group of 224 A $\beta$-negative cognitively normal individuals. ${ }^{16}$ Because there was no predefined cut-off for 
CSF p-tau181, we established a cut-off at $69.46 \mathrm{pg} / \mathrm{ml}$ based on the mean $+(2 \mathrm{x}$ standard deviation) in $200 \mathrm{~A} \beta$-negative cognitively normal individuals from BioFINDER-2 (of whom 111 overlapped with the current sample). Note that cut-offs were only used for the concordance analyses presented in Figure 2 and Supplementary figure 1, with continuous CSF (and plasma) values used in all other statistical models.

\section{Plasma p-tau biomarkers}

Plasma biomarkers were measured as described previously. ${ }^{16}$ Plasma p-tau181 was quantified using an in-house Simoa-based immunoassay at the Clinical Neurochemistry Laboratory in Gothenburg. ${ }^{14}$ Analysis of plasma p-tau 217 was performed at Eli Lilly and Company using the MSD platform. ${ }^{12,15}$ For both plasma p-tau181 and p-tau217 one outlier was removed. Out of 399 study participants, 110 had plasma p-tau217 levels below the detection limit of the assay. When plasma p-tau217 concentrations could not be interpolated from the standard curve due to very low signal, values were imputed to the lowest measurable value. Out of 110 samples below the detection limit, plasma p-tau217 values were imputed for 41 cases $(10.3 \%$ of the total sample). Note that $92.7 \%(38 / 41)$ of imputed data and $92.7 \%(102 / 110)$ of samples below the detection limit were present in the $A \beta$-negative group. Therefore, these values were considered to represent truly very low p-tau217 concentrations and were included in all statistical analysis. Cutoffs for concordance analyses were previously established at 11.9 $\mathrm{pg} / \mathrm{mL}$ for plasma $\mathrm{p}$-tau181 and at $2.5 \mathrm{pg} / \mathrm{mL}$ for plasma $\mathrm{p}$-tau 217 , based on the mean $+(2 \mathrm{x}$ standard deviation) in group of $224 \mathrm{~A} \beta$-negative cognitively normal individuals. ${ }^{16}$

\section{Statistical analyses}

Statistical analysis was performed using R software (Version 4.0.3). When presenting group characteristics, patient features were compared using two samples t-tests and Chi-squared tests. 
Non-adjusted Pearson correlation coefficients were calculated using the corrplot package. We accounted for missing values using multiple imputations using the mice package (25 imputations and 5 iterations), as both of the methods described below needed all values to be present. An overview of the proportion of missing values is presented in Supplementary table 1.

First, we examined the correlations between the different tau biomarkers and AD-related features, as well as the degree of concordance between the PET, CSF and plasma tau biomarkers.

Second, we tested whether CSF p-tau181 and tau PET in the temporal meta-ROI were differentially associated with other AD-related features. As those features, we selected age, $A P O E$ \&4 carriership, amyloid biomarkers (CSF $\mathrm{A} \beta_{42 / 40}$ ratio, amyloid PET global SUVR/Centiloids), cognitive measures (MMSE and composite z-scores for memory, language, executive functioning and visuospatial domains ${ }^{61-63}$ ), and structural MRI measures (hippocampal volumes [adjusted for intracranial volume] and AD-signature cortical thickness). In line with comparable work on amyloid biomarkers ${ }^{81}$, we first created three sets of regularized regression models using ridge regression: (i) AD-related feature predicted by CSF p-tau181 (simple model), (ii) AD-related feature predicted by PET temporal meta-ROI (simple model), (iii) AD-related feature predicted by both CSF p-tau181 and PET temporal meta-ROI (combined model). All models were adjusted for age and sex, and models predicting cognitive performance were additionally adjusted for education. We chose to use ridge regression because it provides stable estimates of $\beta$-coefficients despite correlated predictors ${ }^{82}$, which is the case in our combined models. From this set of analyses, we computed four $95 \%$ confidence intervals $(\mathrm{CI})$ of $\beta$-coefficients for each AD-related feature (i.e., for PET from the simple 
model, for CSF from the simple model, and for both PET and CSF from the combined model) by using bootstrapped sampling with replacement ( $\mathrm{N}=1,000$ iterations). We used these $95 \%$ CIs to compare the $\beta$-coefficients of models for PET and CSF. Note that amyloid PET was not performed in cases with dementia in the BioFINDER-2 study. Hence, amyloid PET SUVR values were not imputed (because it would introduce systematic bias), and the bootstrapped samples were taken only from cases with available amyloid PET. To compare the strength of the associations between PET and CSF tau biomarkers with the predicted AD-features, we followed these three criteria: (i) non-overlapping 95\% CIs of the $\beta$-coefficient of the simple models for CSF and PET (i.e., stronger association for the biomarker with the more positive or negative value), (ii) $95 \%$ CIs in the simple or combined models non-overlapping with $\beta=0$ for only CSF or PET (stronger association for the tau biomarker non-overlapping with $\beta=0$, (iii) a significant drop of the $\beta$-coefficient from the combined model relative to the simple model for only CSF or PET (i.e., overlapping 95\% CIs for one tau biomarker between the simple and combined model, but non-overlapping 95\% CIs for the other, with a stronger association for the biomarker with overlapping 95\% CIs). In case a feature was not significantly associated with both tau biomarkers, we did not perform the combined model. We also investigated whether the results were similar when comparing the change in percentage of $\beta$-coefficients from the simple to the combined model, and when comparing the R-squared values between the simple models of CSF and PET. After the primary analysis in BioFINDER-2, we aimed to replicate the findings using the ADNI dataset.

Third, to identify the AD-features that were most strongly associated with the tau biomarkers, we created parallel regression tree models (predicting CSF p-tau181 levels and tau PET SUVR in the temporal meta-ROI) with continuous $\mathrm{AD}$-related patient features using the caret package. These regression trees provide an estimate of relative strength of the association 
between $\mathrm{AD}$-related patient features and tau biomarkers, while adjusting for all other variables in the model ${ }^{83}$ Regression tree models are based on binary recursive partitioning in which each fork is a split of a predictor variable and each node at the end has a prediction for the continuous outcome variable. The splits are based on minimizing the overall sums of squares error and are pruned down to reduce over-fitting. We used bootstrap aggregation (i.e., bagging) with $\mathrm{N}=25$ bootstrap replications in which final predictions are based on the average of the replications. Model accuracy is tested using 10 -fold cross-validation. We used the variable importance measure (VIM) from the models to estimate the relative importance of variables to predict tau biomarkers. The VIM for a variable is based on the average decrease of root mean squared error when it is used in the model, and higher VIM indicates that the variable is considered more important to predict the outcome. In our analysis, we present the VIMs from N=25 bagged regression tree models, created with each of the 25 imputed datasets.

Fourth, we aimed to explore p-tau181 in plasma, a different p-tau isoform (i.e., p-tau217) in plasma and CSF, and an earlier affected tau PET region (i.e., entorhinal cortex) in the BioFINDER-2 cohort only. Therefore, we conducted ridge regression models (as described above) to investigate the potentially different associations with AD-features using different pairings of tau biomarkers, i.e., (i) tau PET SUVR in the temporal meta-ROI vs plasma ptau181 levels, (ii) tau PET SUVR in the temporal meta-ROI vs plasma and CSF p-tau217 levels, and (iii) tau PET SUVR in the entorhinal cortex vs CSF p-tau181.

\section{Funding}

This project has received funding from the European Research Council (949570), the Swedish Research Council (2016-00906), the Knut and Alice Wallenberg foundation (2017-0383), the Marianne and Marcus Wallenberg foundation (2015.0125), the Strategic Research Area 
MultiPark (Multidisciplinary Research in Parkinson's disease) at Lund University, the Swedish Alzheimer Foundation (AF-939932), the Swedish Brain Foundation (FO2019-0326), The Parkinson foundation of Sweden (1280/20), the Skåne University Hospital Foundation (2020O000028), Regionalt Forskningsstöd (2020-0314) and the Swedish federal government under the ALF agreement (2018-Projekt0279). The precursor of ${ }^{18} \mathrm{~F}$-flutemetamol was provided by GE Healthcare in BioFINDER-2 and the precursor of ${ }^{18} \mathrm{~F}-\mathrm{RO} 948$ was provided by Roche in BioFINDER-2. HZ is a Wallenberg Scholar. KB is supported by the Swedish Research Council (\#2017-00915), the Swedish Alzheimer Foundation (\#AF-742881), Hjärnfonden, Sweden (\#FO2017-0243), and the Swedish state under the agreement between the Swedish government and the County Councils, the ALF-agreement (\#ALFGBG-715986).

Data collection and sharing for this project was funded by the Alzheimer's Disease Neuroimaging Initiative (ADNI) (National Institutes of Health Grant U01 AG024904) and DOD ADNI (Department of Defense award number W81XWH-12-2-0012). ADNI is funded by the National Institute on Aging, the National Institute of Biomedical Imaging and Bioengineering, and through generous contributions from the following: AbbVie, Alzheimer's Association; Alzheimer's Drug Discovery Foundation; Araclon Biotech; BioClinica, Inc.; Biogen; Bristol-Myers Squibb Company; CereSpir, Inc.; Cogstate; Eisai Inc.; Elan Pharmaceuticals, Inc.; Eli Lilly and Company; EuroImmun; F. Hoffmann-La Roche Ltd and its affiliated company Genentech, Inc.; Fujirebio; GE Healthcare; IXICO Ltd.; Janssen Alzheimer Immunotherapy Research \& Development, LLC.; Johnson \& Johnson Pharmaceutical Research \& Development LLC.; Lumosity; Lundbeck; Merck \& Co., Inc.; Meso Scale Diagnostics, LLC.; NeuroRx Research; Neurotrack Technologies; Novartis Pharmaceuticals Corporation; Pfizer Inc.; Piramal Imaging; Servier; Takeda Pharmaceutical Company; and Transition Therapeutics. The Canadian Institutes of Health Research is 
providing funds to support ADNI clinical sites in Canada. Private sector contributions are facilitated by the Foundation for the National Institutes of Health (www.fnih.org). The grantee organization is the Northern California Institute for Research and Education, and the study is coordinated by the Alzheimer's Therapeutic Research Institute at the University of Southern California. ADNI data are disseminated by the Laboratory for Neuro Imaging at the University of Southern California.

\section{Conflicts of interest/Competing interests}

HZ has served at scientific advisory boards for Eisai, Denali, Roche Diagnostics, Wave, Samumed, Siemens Healthineers, Pinteon Therapeutics, Nervgen, AZTherapies and CogRx, has given lectures in symposia sponsored by Cellectricon, Fujirebio, Alzecure and Biogen, and is a co-founder of Brain Biomarker Solutions in Gothenburg AB (BBS), which is a part of the GU Ventures Incubator Program. KB has served as a consultant, at advisory boards, or at data monitoring committees for Abcam, Axon, Biogen, JOMDD/Shimadzu. Julius Clinical, Lilly, MagQu, Novartis, Roche Diagnostics, and Siemens Healthineers, and is a co-founder of Brain Biomarker Solutions in Gothenburg AB (BBS), which is a part of the GU Ventures Incubator Program. SP has served at scientific advisory boards for Roche and Geras Solutions. JLD is an employee and shareholder of Eli Lilly and Company. $\mathrm{OH}$ has acquired research support (for the institution) from AVID Radiopharmaceuticals, Biogen, Eli Lilly, Eisai, GE Healthcare, Pfizer, and Roche. In the past 2 years, he has received consultancy/speaker fees from AC Immune, Alzpath, Biogen, Cerveau and Roche.

The other authors report no conflicts of interest 
Table 1. Participant characteristics

BioFINDER-2

ADN

\begin{tabular}{rcccccc} 
& Total & CU & MCI/Dem & Total & CU & MCI/Dem \\
\cline { 2 - 7 } & 400 & 219 & 181 & 371 & 242 & 129 \\
Age & $67.7(11.4)$ & $64.57(12.8)$ & $71.5(7.8)^{*}$ & $73.2(7.7)$ & $72.7(7.2)$ & $74.3(8.5)^{*}$ \\
Sex, female (\%) & $195(49)$ & $104(47)$ & $91(50)$ & $206(56)$ & $149(62)$ & $57(44)^{*}$ \\
Dementia (\%) & $85(21)$ & $0(0)$ & $85(47)$ & $32(9)$ & $0(0)$ & $32(25)$ \\
Education, years & $12.7(4.0)$ & $12.8(3.5)$ & $12.6(4.5)$ & $16.6(2.4)$ & $16.9(2.2)$ & $16.1(2.6)^{*}$ \\
$\varepsilon 4$ carriership (\%) & $211(53)$ & $97(44)$ & $114(63)^{*}$ & $145(39)$ & $88(36)$ & $57(44)$
\end{tabular}

\section{Tau biomarkers:}

Plasma p-tau181

Plasma p-tau217

CSF p-tau181

CSF p-tau 217

$114(63)^{*}$

$145(39)$

$88(36)$

$57(44)$

Tau PET entorhinal

$8.1(5.58)$

$3.0(3.68)$

$94.8(87.4)$

$219.9(281.2)$

$6.3(4.5)$

$1.3(1.6)$

$55.6(39.9)$

$1.40(0.45)$

$88.1(106.5)$

$10.3(6.0)^{*}$

$5.0(4.4)^{*}$

$1.42(0.54)$

$1.16(0.19)$

$142.2(104.4) *$

379.4 (338.9)*

$1.69(0.50)^{*}$

$24.5(12.8)$

$4.5(12.8)$

-

Tau PET temporal meta-RO

$1.18(0.17)$

$1.71(0.67)^{*}$

$1.21(0.22)$

$22.2(10.7)$

$-$

$28.9(15.2)^{*}$

$1.26(0.26)$

$1.15(0.14)$

$1.20(0.13)$

$1.33(0.28)^{*}$

AD-features:

CSF A $\beta_{42 / 40}$ Amyloid PET SUVR / CL

$0.78(0.31)$

$0.92(0.27)$

$0.62(0.28)^{*}$

$0.06(0.03)$

$0.07(0.02)$ $1.39(0.37)^{*}$

$0.73(0.20)$

$0.68(0.15)$

$0.84(0.23)^{*}$

$34.3(43.1)$

$25.6(35.5)$

$0.05(0.03)^{*}$

$28.9(1.2)$

$-1.14(1.53)$

$-0.12(0.88)$

$23.7(4.7)^{*}$

$28.2(2.4)$

$-0.12(0.86)$

$-2.44(1.14)^{*}$

$0.70(0.78)$

$29.1(1.3)$

$-0.91(1.46)$

$-0.18(0.77)$

$-1.88(1.47)^{*}$

$0.65(0.90)$

$1.05(0.56)$

$50.7(50.8) *$

Language composite $z$-score

$-0.84(2.55)$

$-0.04(0.72)$

$-1.88(3.54)^{*}$

$0.81(1.03)$

$0.97(0.75)$

$26.5(3.1)^{*}$

Visuospatial composite $\mathrm{z}$-score
Visuctioning composite

$2.28(0.37)$

$2.45(0.29)$

$2.09(0.36)^{*}$

$0.05(0.85)^{*}$

MRI Hippocampal volume / TIV ratio

$\begin{array}{ccc}\text { MRI AD signature region thickness } & 2.61(0.22) & 2.72(0.16) \\ \text { Participant characteristics for the discovery cohort (i.e., BioFINDER-2) and the validation cohort (i.e., }\end{array}$

$2.72(0.16)$

$2.48(0.21)^{*}$

$2.47(0.37)$

$2.58(0.31)$

$-0.19(0.90) *$

$<0.05)$ between the cognitively unimpaired $(\mathrm{CU})$ and the symptomatic (including mild cognitive impairment and dementia) groups are marked with an asterisk in the MCI/Dem group column. Several values are not directly comparable between the discovery cohort and validation cohort, including (i) CSF p-tau181 (assays differ), (ii) tau PET (RO948 in BioFINDER-2, flortaucipir in ADNI), (iii) CSF A $\beta$ 42/40 (assays differ), (iv) amyloid PET (SUVR for BioFINDER-2, Centiloids for ADNI, (v) cognitive test composite scores (composed of different tests and standardization differed. MRI Hippocampal volume / TIV ratio values are multiplied by 1000. 
bioRxiv preprint doi: https://doi.org/10.1101/2021.04.19.440402; this version posted April 19, 2021. The copyright holder for this preprint (which was not certified by peer review) is the author/funder, who has granted bioRxiv a license to display the preprint in perpetuity. It is made available under aCC-BY-ND 4.0 International license.

Figure 1. Correlations between tau biomarkers and other AD-related features

A. BioFINDER-2
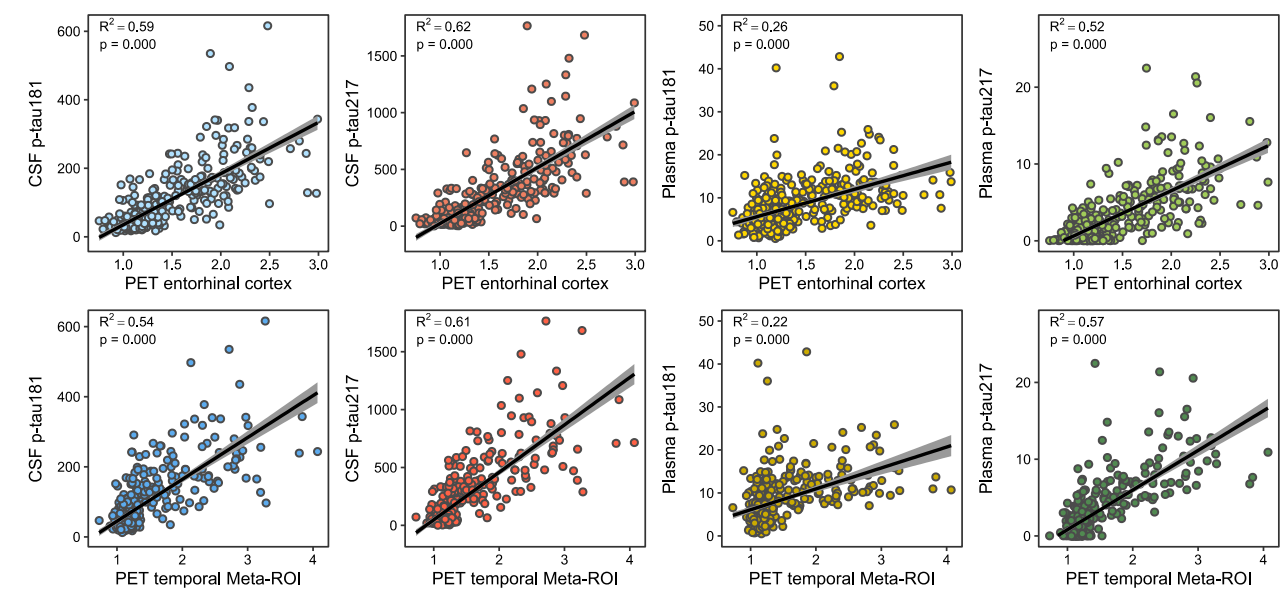

\section{B. BioFINDER-2}

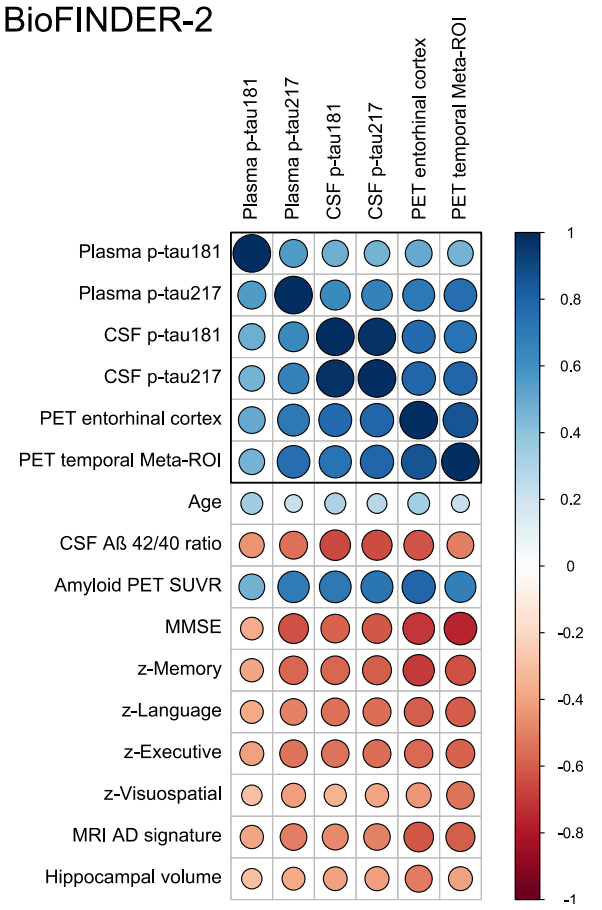

C. ADNI

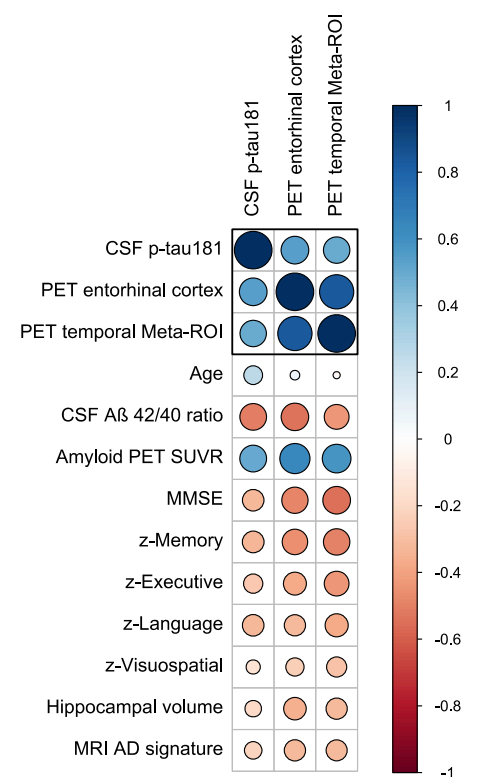

Graphs display the correlations between the tau PET/fluid biomarkers and AD features in BioFINDER-2 (A and B) and ADNI (C). A) Scatterplots of the associations between tau PET uptake in the entorhinal cortex (upper panel) and in the temporal meta-ROI (lower panel) with from left to right CSF p-tau181, CSF p-tau217, plasma p-tau181 and plasma p-tau217. B) [BioFINDER-2] and C) [ADNI] showing matrices of correlation coefficients between all available tau biomarkers and AD-related features. Strong positive correlations are indicated in dark blue, while strong negative correlations are indicated in red. 
Figure 2. Concordance between tau PET in the temporal meta-ROI and CSF and plasma p-tau181 and p-tau217 in BioFINDER-2
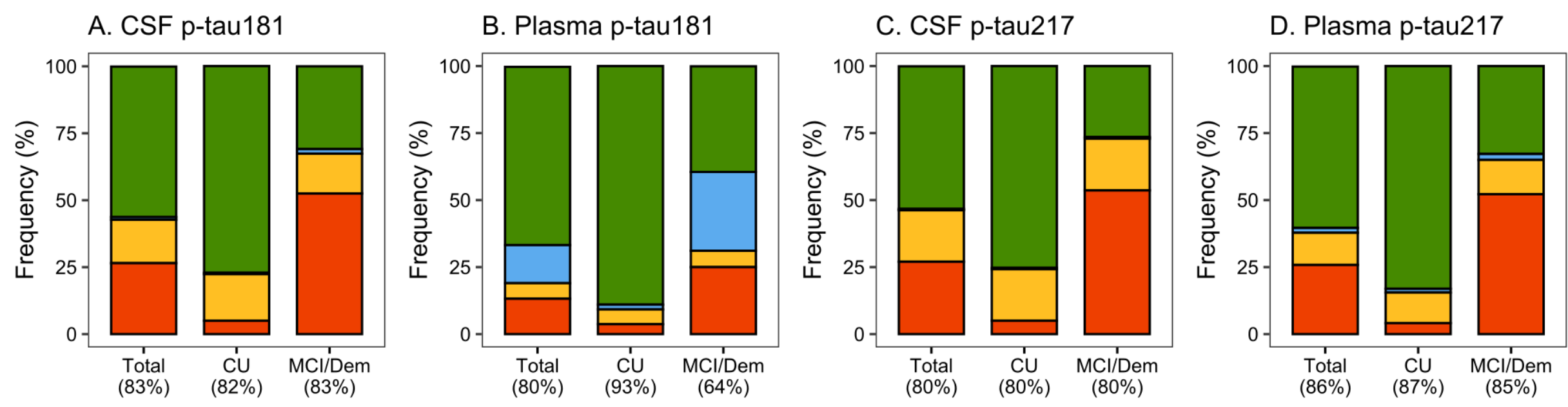

Fluid-/PET-

Fluid-/PET+

Fluid+/PET-

Fluid+/PET+

The graphs represent concordance rates between the different tau biomarkers in BioFINDER-2. A) Tau PET in the temporal Meta-ROI vs CSF p-tau181, B) Tau PET in the temporal meta-ROI vs plasma p-tau181, C) Tau PET in the temporal meta-ROI vs CSF p-tau217, and D) Tau PET in the temporal meta-ROI vs plasma p-tau217. Green indicates negative concordance, red indicates positive concordance, blue indicates discordance where tau PET is positive with negative fluid biomarkers, and orange indicates discordance where tau PET is negative with positive fluid biomarkers. Cut-offs for both tau PET and fluid biomarkers are based on the mean $+(2 \mathrm{x}$ standard deviation) in A $\beta$ negative cognitively normal individuals (see Methods section for further detail). 
bioRxiv preprint doi: https://doi.org/10.1101/2021.04.19.440402; this version posted April 19, 2021. The copyright holder for this preprint (which was not certified by peer review) is the author/funder, who has granted bioRxiv a license to display the preprint in perpetuity. It is made available under aCC-BY-ND 4.0 International license.

Figure 3. Differential associations of tau PET vs CSF p-tau181 with Alzheimer related features

\section{A. Discovery cohort (BioFINDER-2)}
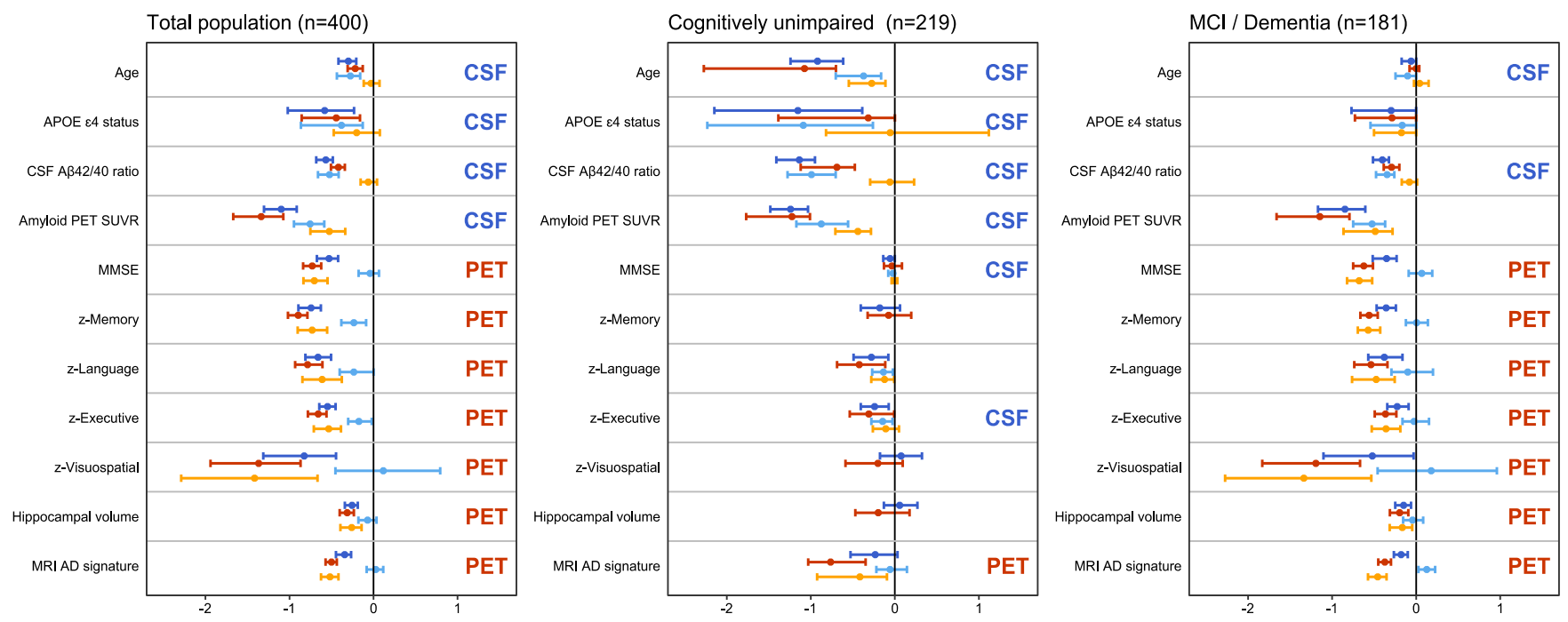

\section{B. Replication cohort (ADNI)}
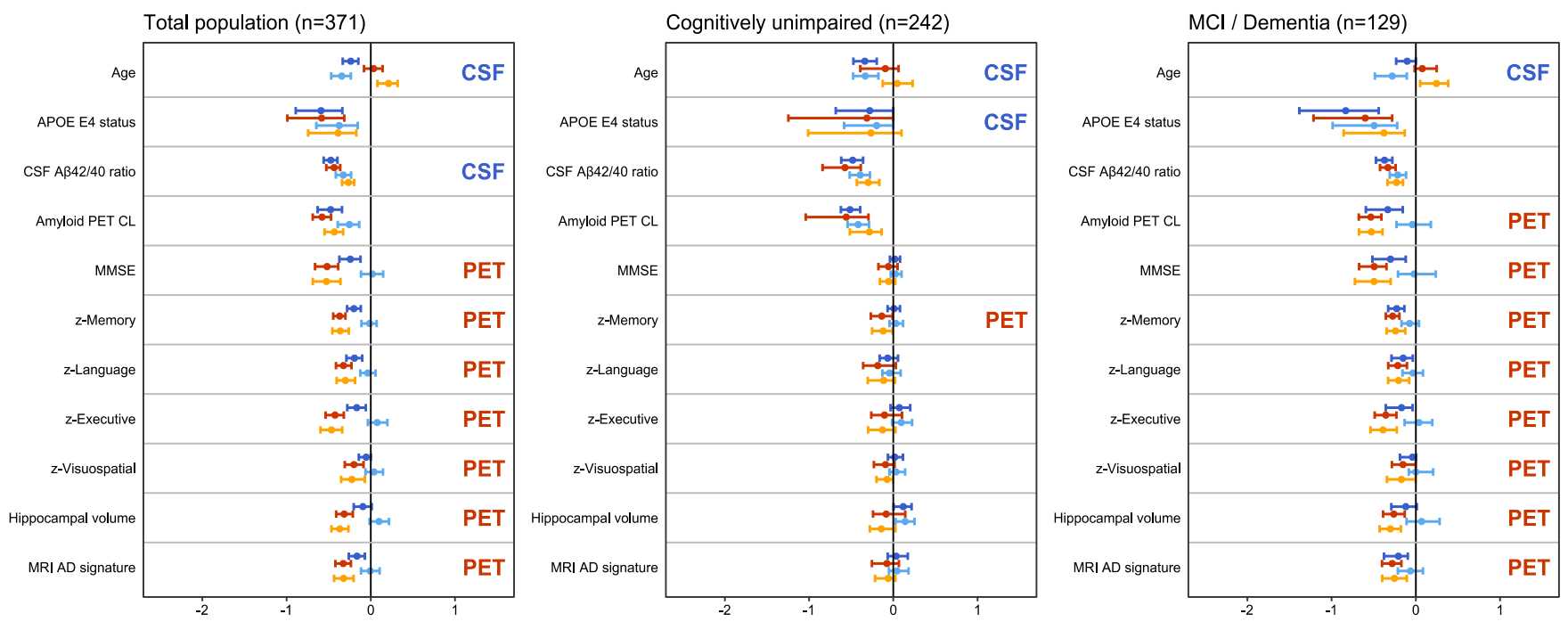

$\rightarrow \operatorname{CSF} \beta \rightarrow$ PET $\beta \longrightarrow \operatorname{CSF} \beta$ (combined model) $\multimap$ PET $\beta$ (combined model)

Graphs display the differential association of temporal Meta-ROI tau PET vs CSF p-tau181 with AD-related features in the discovery cohort BioFINDER-2 (panel A) and the validation cohort ADNI (panel B). Median and $95 \%$ confidence intervals $(\mathrm{CI})$ of $\beta$-coefficients are plotted from the following ridge regression models: (i) ADrelated feature predicted by CSF p-tau181 (dark blue, simple model), (ii) AD-related feature predicted by tau PET (dark red, simple model), (iii) AD-related feature predicted by the combination of CSF p-tau181 (light blue) and tau PET (orange, combined model). In case a feature was non-significant for both tau biomarkers in the simple models (i.e., 95\% CIs crossed the 0 -line), no combined model was performed. All models were adjusted for age and sex, and cognitive tests were additionally adjusted for education. The $\beta$-coefficients for age, APOE $\varepsilon 4$ carriership and amyloid PET global measures were multiplied by -1 for visualisation purposes.

Three criteria were used to determine whether one biomarker showed a stronger association with an AD feature compared to the other tau biomarker: (i) the $95 \%$ CI between the simple models are non-overlapping (stronger association for the biomarker with more extreme values), (ii) the $95 \% \mathrm{CI}$ in the simple or combined models cross the 0-line (stronger association for the biomarker that did not cross the 0-line), and (iii) there is a lack of a significant drop in the $\beta$-coefficient from the combined model relative to the simple model for one of the two tau biomarkers, reflected by overlapping $95 \%$ CIs between the simple and combined model for one tau biomarker in the presence of non-overlapping 95\% CIs for the other tau biomarker (stronger association for the biomarker with overlapping $95 \%$ CIs). If one or more of these criteria were met, this was marked on the right side of plots. 
Figure 4. Rank-ordering of importance in predicting CSF p-tau181 and tau PET levels

\section{A. Discovery cohort (BioFINDER-2)}
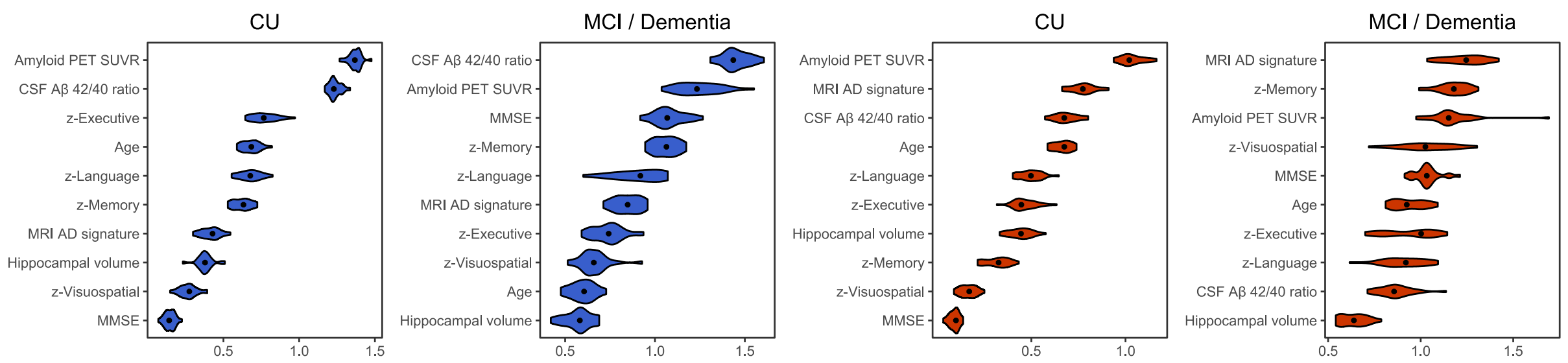

B. Replication cohort (ADNI)
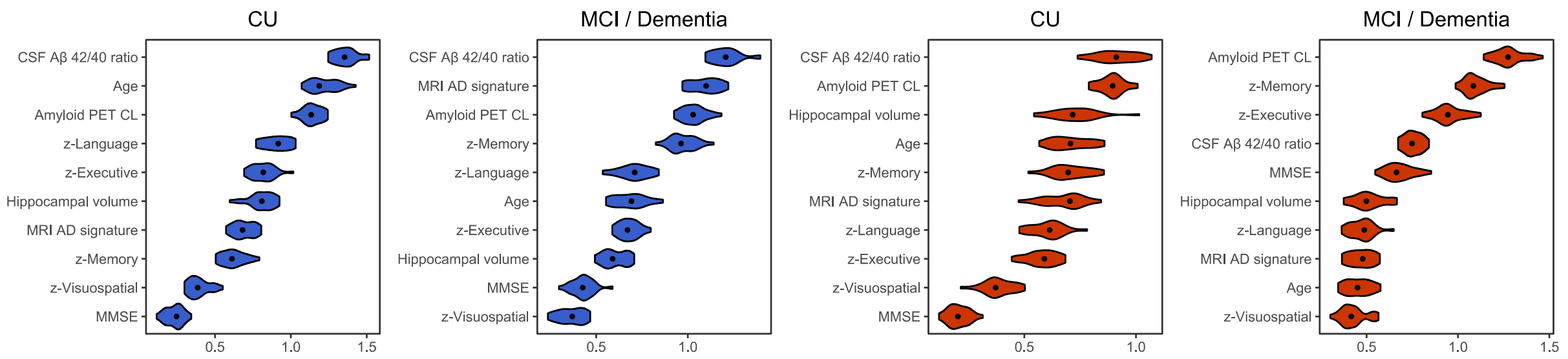

- CSF p-tau181 $\bullet$ Tau PET temporal Meta-ROI

Graphs shows in a rank-ordered manner the most important predictors of CSF p-tau181 (blue) and tau PET temporal meta-ROI (red) levels in BioFINDER-2 (A) and ADNI

(B). The violin plots of the variable importance measures (VIM) are based on the computation of 25 times the regression tree models using multiple imputation datasets (25x). Median VIM values are marked with a dot. 
bioRxiv preprint doi: https://doi.org/10.1101/2021.04.19.440402; this version posted April 19, 2021. The copyright holder for this preprint (which was not certified by peer review) is the author/funder, who has granted bioRxiv a license to display the preprint in perpetuity. It is made available under aCC-BY-ND 4.0 International license.

Figure 5. Differential associations of tau PET vs plasma p-tau181, plasma p-tau217 and CSF p-tau217 with Alzheimer related features in BioFINDER-2

A. Tau PET vs plasma $p$-tau 181
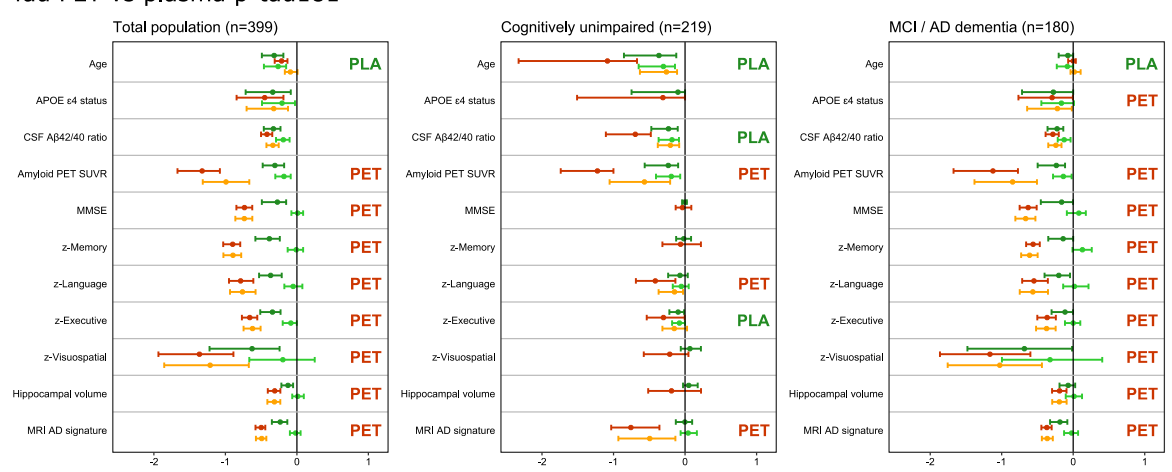

B. Tau PET vs plasma p-tau217
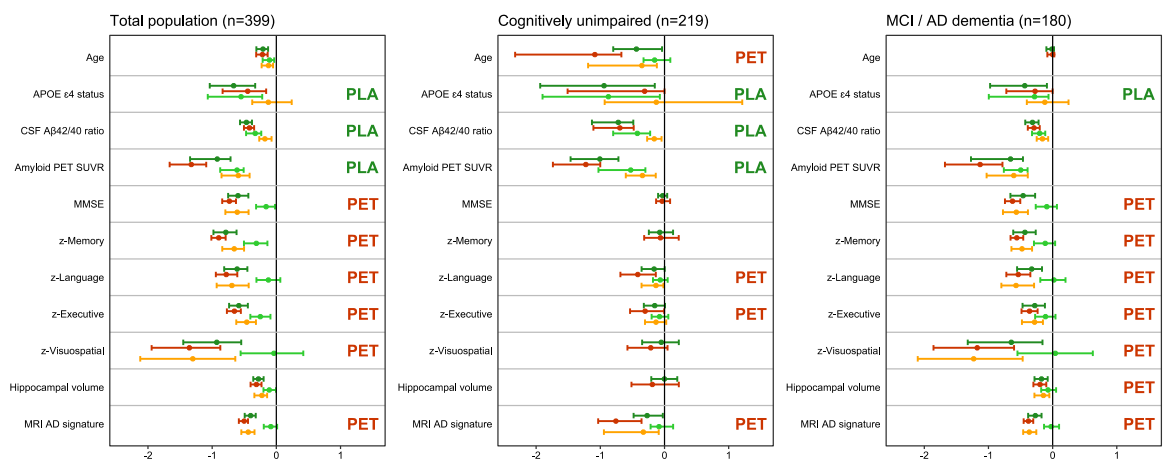

C. Tau PET vs CSF p-tau217

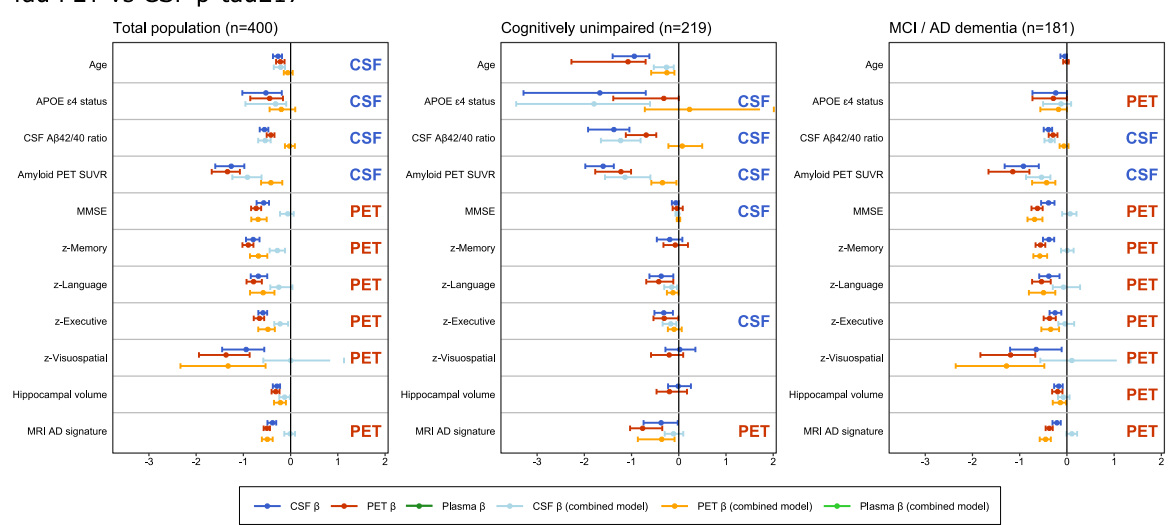

Graphs display the differential association of temporal meta-ROI tau PET vs plasma p-tau181 (panel A), vs plasma p-tau217 (panel B) and vs CSF p-tau217 (panel C) with AD-related features in BioFINDER-2. Median and 95\% confidence intervals $(\mathrm{CI})$ of $\beta$-coefficients are plotted from the following ridge regression models: (i) AD-related feature predicted by fluid biomarkers (dark blue, simple model), (ii) AD-related feature predicted by tau PET (dark red, simple model), (iii) AD-related feature predicted by the combination of fluid biomarkers (light blue) and tau PET (orange, combined model). In case a feature was non-significant for both tau biomarkers in the simple models (i.e., $95 \%$ CIs crossed the 0 -line), no combined model was performed. All models were adjusted for age and sex, and cognitive tests were additionally adjusted for education. The $\beta$-coefficients for age, APOE $\varepsilon 4$ carriership and amyloid PET global measures were multiplied by -1 for visualisation purposes.

Three criteria were used to determine whether one biomarker showed a stronger association with an AD feature compared to the other tau biomarker: (i) the $95 \%$ CI between the simple models are non-overlapping (stronger association for the biomarker with more extreme values), (ii) the $95 \% \mathrm{CI}$ in the simple or combined models cross the 0-line (stronger association for the biomarker that did not cross the 0-line), and (iii) there is a lack of a significant drop in the $\beta$-coefficient from the combined model relative to the simple model for one of the two tau biomarkers, reflected by overlapping $95 \%$ CIs between the simple and combined model for one tau biomarker in the presence of non-overlapping $95 \%$ CIs for the other tau biomarker (stronger association for the biomarker with overlapping $95 \% \mathrm{CIs}$ ). If one or more of these criteria were met, this was marked on the right side of plots. 


\section{SUPPLEMENTARY MATERIAL}

Supplementary table 1. Proportion of missing values

BioFINDER-2 (n=404) ADNI (n=371)

\begin{tabular}{lcc}
\hline Education, years & $1(0.2)$ & $0(0)$ \\
APOE \&4 carriership & $0(0)$ & $0(0)$ \\
Plasma p-tau181 & $1(0.2)$ & - \\
Plasma p-tau217 & $1(0.2)$ & - \\
CSF p-tau181 & $0(0)$ & $0(0)$ \\
CSF p-tau217 & $0(0)$ & $0(0)$ \\
CSF t-tau & & $0(0)$ \\
Tau PET & $0(0)$ & \\
& & $33(8.9)$ \\
CSF A $\beta$ 42/40 & $0(0)$ & $1(0.3)$ \\
Amyloid PET & $83(20.8)$ & $0(0)$ \\
MMSE & $0(0)$ & $1(0.3)$ \\
Memory composite & $11(2.8)$ & $0(0)$ \\
Language composite & $7(1.8)$ & $0(0)$ \\
Executive functioning composite & $3(0.8)$ & $0(0)$ \\
Visuospatial composite & $20(5.0)$ & $7(1.9)$ \\
MRI Hippocampal volume/ TIV ratio & $9(2.2)$ & $17(4.6)$ \\
MRI AD signature region thickness & $9(2.2)$ & \\
\hline
\end{tabular}

Missing values are presented as n (\%)

$\mathrm{TIV}=$ Total intracranial volume 
Supplementary table 2. Percentage of $\beta$-coefficient change between the simple and combined model in BioFINDER-2

\begin{tabular}{|c|c|c|c|c|c|c|c|c|c|}
\hline \multirow[b]{2}{*}{ Feature } & \multicolumn{3}{|l|}{ Total } & \multicolumn{3}{|l|}{$\mathbf{C U}$} & \multicolumn{3}{|l|}{ MCI/Dem } \\
\hline & PET & CSF & & PET & CSF & & PET & CSF & \\
\hline Age & $\begin{array}{c}-86 \\
(-147,-57)\end{array}$ & $\begin{array}{c}-8 \\
(-36,20)\end{array}$ & $*$ & $\begin{array}{c}-75 \\
(-88,-60)\end{array}$ & $\begin{array}{c}-58 \\
(-80,-35)\end{array}$ & & $\begin{array}{c}-83 \\
(-139353,162532)\end{array}$ & $\begin{array}{c}48 \\
(-96,10655)\end{array}$ & \\
\hline$A P O E \varepsilon 4$ carriership & $\begin{array}{c}-53 \\
(-127,-34)\end{array}$ & $\begin{array}{c}-35 \\
(-55,16)\end{array}$ & & $\begin{array}{c}-68 \\
(-105110,15928)\end{array}$ & $\begin{array}{c}-8 \\
(-47,56)\end{array}$ & & $\begin{array}{c}-38 \\
(-87,56)\end{array}$ & $\begin{array}{c}-42 \\
(-97,214)\end{array}$ & \\
\hline $\mathrm{CSF} A \beta_{42 / 40}$ & $\begin{array}{c}-85 \\
(-110,-67)\end{array}$ & $\begin{array}{c}-8 \\
(-20,6)\end{array}$ & $*$ & $\begin{array}{c}-92 \\
(-143,-71)\end{array}$ & $\begin{array}{c}-14 \\
(-38,6)\end{array}$ & $*$ & $\begin{array}{c}-71 \\
(-105,-52)\end{array}$ & $\begin{array}{c}-14 \\
(-28,4)\end{array}$ & $*$ \\
\hline Amyloid PET SUVR & $\begin{array}{c}-61 \\
(-72,-50)\end{array}$ & $\begin{array}{c}-31 \\
(-42,-21)\end{array}$ & $*$ & $\begin{array}{c}-64 \\
(-76,-52)\end{array}$ & $\begin{array}{c}-29 \\
(-53,-14)\end{array}$ & & $\begin{array}{c}-57 \\
(-70,-41)\end{array}$ & $\begin{array}{c}-38 \\
(-49,-26)\end{array}$ & \\
\hline MMSE & $\begin{array}{c}-4 \\
(-19,7)\end{array}$ & $\begin{array}{c}-92 \\
(-115,-70)\end{array}$ & $*$ & $\begin{array}{c}-81 \\
(-159,59)\end{array}$ & $\begin{array}{c}-58 \\
(-82,-22)\end{array}$ & & $\begin{array}{c}8 \\
(-8,24)\end{array}$ & $\begin{array}{c}-118 \\
(-176,-80)\end{array}$ & $*$ \\
\hline Memory composite & $\begin{array}{c}-18 \\
(-34,-6)\end{array}$ & $\begin{array}{c}-69 \\
(-87,-52)\end{array}$ & $*$ & $\begin{array}{c}-78 \\
(-466,343)\end{array}$ & $\begin{array}{c}-31 \\
(-97,29)\end{array}$ & & $\begin{array}{c}1 \\
(-16,18)\end{array}$ & $\begin{array}{c}-101 \\
(-156,-71)\end{array}$ & $*$ \\
\hline Language composite & $\begin{array}{c}-22 \\
(-42,0)\end{array}$ & $\begin{array}{c}-65 \\
(-100,-44)\end{array}$ & $*$ & $\begin{array}{c}-71 \\
(-91,-48)\end{array}$ & $\begin{array}{c}-51 \\
(-71,-32)\end{array}$ & & $\begin{array}{c}-11 \\
(-42,27)\end{array}$ & $\begin{array}{c}-74 \\
(-199,-41)\end{array}$ & \\
\hline Executive functioning composite & $\begin{array}{c}-19 \\
(-37,-1)\end{array}$ & $\begin{array}{c}-68 \\
(-96,-50)\end{array}$ & $*$ & $\begin{array}{c}-65 \\
(-122,-30)\end{array}$ & $\begin{array}{c}-39 \\
(-69,-14)\end{array}$ & & $\begin{array}{c}-3 \\
(-36,31)\end{array}$ & $\begin{array}{c}-89 \\
(-220,-46)\end{array}$ & $*$ \\
\hline Visuospatial composite & $\begin{array}{c}6 \\
(-38,38)\end{array}$ & $\begin{array}{c}-114 \\
(-232,-55)\end{array}$ & $*$ & $\begin{array}{c}-32 \\
(-99,131)\end{array}$ & $\begin{array}{c}-27 \\
(-1360,1175)\end{array}$ & & $\begin{array}{c}12 \\
(-36,60)\end{array}$ & $\begin{array}{c}-133 \\
(-2729,-47)\end{array}$ & * \\
\hline MRI Hippocampal volume/ TIV ratio & $\begin{array}{c}-17 \\
(-44,7)\end{array}$ & $\begin{array}{c}-72 \\
(-116,-40)\end{array}$ & & $\begin{array}{c}-20 \\
(-263,198)\end{array}$ & $\begin{array}{c}-6 \\
(-894,724)\end{array}$ & & $\begin{array}{c}-15 \\
(-63,29)\end{array}$ & $\begin{array}{c}-69 \\
(-203,-18)\end{array}$ & \\
\hline MRI AD signature cortical thickness & $\begin{array}{c}4 \\
(-11,18)\end{array}$ & $\begin{array}{c}-108 \\
(-140,-80)\end{array}$ & $*$ & $\begin{array}{c}-44 \\
(-79,1)\end{array}$ & $\begin{array}{c}-67 \\
(-252,52)\end{array}$ & & $\begin{array}{c}22 \\
(5,44)\end{array}$ & $\begin{array}{c}-172 \\
(-292,-112)\end{array}$ & * \\
\hline
\end{tabular}

Data are presented as the median (95\% confidence interval [CI]) of the change in percentage of the $\beta$-coefficient between the simple and the combined model for both tau PET and CSF ptau-181. Values were taken from the ridge regression models over 1000 bootstrapped samples. Asterisks and bold text mark the cases where the $95 \%$ CI between temporal meta-ROI tau PET and CSF p-tau181 models did not overlap, indicating that there is a greater association between the patient feature and the modality that has a smaller decrease in $\beta$-coefficient. 
Supplementary table 3. Difference of R-squared between ridge regression models for tau PET vs CSF p-tau181 in BioFINDER-2

\begin{tabular}{|c|c|c|c|c|c|c|c|c|c|}
\hline \multirow[b]{2}{*}{ Feature } & \multicolumn{3}{|l|}{ Total } & \multicolumn{3}{|l|}{$\mathbf{C U}$} & \multicolumn{3}{|l|}{ MCI / Dem } \\
\hline & PET & CSF & & PET & & & PET & CSF & \\
\hline Age & $0.06(0.03,0.10)$ & $0.11(0.07,0.16)$ & & $0.14(0.09,0.23)$ & $0.16(0.10,0.23)$ & & $0.02(0.00,0.08)$ & $0.04(0.00,0.12)$ & \\
\hline CSF $\mathrm{A} \beta_{42 / 40}$ & $0.37(0.31,0.43)$ & $0.50(0.45,0.55)$ & $*$ & $0.22(0.14,0.30)$ & $0.47(0.38,0.55)$ & $*$ & $0.29(0.19,0.39)$ & $0.41(0.32,0.49)$ & \\
\hline Amyloid PET SUVR & $0.47(0.39,0.55)$ & $0.57(0.49,0.65)$ & & $0.39(0.25,0.51)$ & $0.61(0.49,0.70)$ & & $0.47(0.34,0.62)$ & $0.47(0.35,0.62)$ & \\
\hline MMSE & $0.59(0.51,0.68)$ & $0.36(0.28,0.44)$ & $*$ & $0.08(0.02,0.17)$ & $0.09(0.03,0.18)$ & & $0.54(0.43,0.64)$ & $0.23(0.13,0.34)$ & * \\
\hline Memory composite & $0.55(0.48,0.61)$ & $0.45(0.38,0.51)$ & & $0.30(0.21,0.40)$ & $0.31(0.21,0.40)$ & & $0.51(0.41,0.60)$ & $0.30(0.17,0.41)$ & \\
\hline Language composite & $0.46(0.37,0.54)$ & $0.38(0.29,0.46)$ & & $0.25(0.17,0.34)$ & $0.25(0.17,0.34)$ & & $0.32(0.19,0.44)$ & $0.20(0.09,0.33)$ & \\
\hline Executive functioning composite & $0.48(0.40,0.55)$ & $0.39(0.32,0.47)$ & & $0.39(0.29,0.48)$ & $0.39(0.30,0.49)$ & & $0.25(0.14,0.35)$ & $0.13(0.05,0.22)$ & \\
\hline Visuospatial composite & $0.30(0.19,0.43)$ & $0.15(0.07,0.24)$ & & $0.08(0.02,0.16)$ & $0.07(0.01,0.14)$ & & $0.23(0.12,0.36)$ & $0.08(0.00,0.18)$ & \\
\hline MRI Hippocampal volume/ TIV ratio & $0.42(0.36,0.49)$ & $0.39(0.33,0.46)$ & & $0.37(0.28,0.47)$ & $0.37(0.28,0.47)$ & & $0.27(0.17,0.39)$ & $0.24(0.14,0.34)$ & \\
\hline MRI AD signature region thickness & $0.52(0.46,0.58)$ & $0.39(0.33,0.45)$ & $*$ & $0.36(0.23,0.50)$ & $0.28(0.19,0.39)$ & & $0.46(0.35,0.56)$ & $0.27(0.16,0.39)$ & \\
\hline
\end{tabular}

Data are presented as the median (95\% confidence interval [CI]) of R-squared values from simple models for tau PET in the temporal meta-ROI and CSF p-tau181. Values were taken from the ridge regression models over 1000 bootstrapped samples. Asterisks and mark cases where the 95\% CI between PET and CSF models do not overlap, indicating that there is a greater association between the patient feature and the modality that has the bigger R-squared value (also marked by bold). 
Supplementary table 4. R-squared values for simple models for CSF p-tau181 and CSF p-tau217 in BioFINDER-2

\begin{tabular}{|c|c|c|c|c|c|c|}
\hline \multirow[b]{2}{*}{ Feature } & \multicolumn{2}{|l|}{ Total } & \multicolumn{2}{|l|}{$\mathbf{C U}$} & \multicolumn{2}{|l|}{ MCI / Dem } \\
\hline & CSF p-tau181 & CSF p-tau217 & CSF p-tau181 & CSF p-tau217 & CSF p-tau181 & CSF p-tau217 \\
\hline Age & $0.11(0.07,0.16)$ & $0.09(0.05,0.13)$ & $0.16(0.10,0.23)$ & $0.12(0.07,0.19)$ & $0.04(0.00,0.12)$ & $0.03(0.00,0.10)$ \\
\hline $\mathrm{CSF} A \beta_{42 / 40}$ & $0.50(0.45,0.55)$ & $0.49(0.44,0.54)$ & $0.47(0.38,0.55)$ & $0.49(0.42,0.58)$ & $0.41(0.32,0.49)$ & $0.40(0.32,0.48)$ \\
\hline Amyloid PET SUVR & $0.57(0.49,0.65)$ & $0.62(0.52,0.71)$ & $0.61(0.49,0.70)$ & $0.71(0.62,0.77)$ & $0.47(0.35,0.62)$ & $0.49(0.35,0.65)$ \\
\hline MMSE & $0.36(0.28,0.44)$ & $0.40(0.33,0.48)$ & $0.09(0.03,0.18)$ & $0.09(0.03,0.18)$ & $0.23(0.13,0.34)$ & $0.26(0.16,0.37)$ \\
\hline Memory composite & $0.45(0.38,0.51)$ & $0.48(0.41,0.54)$ & $0.31(0.21,0.40)$ & $0.30(0.22,0.40)$ & $0.30(0.17,0.41)$ & $0.32(0.20,0.44)$ \\
\hline Language composite & $0.38(0.29,0.46)$ & $0.40(0.30,0.48)$ & $0.25(0.17,0.34)$ & $0.25(0.17,0.35)$ & $0.20(0.09,0.33)$ & $0.21(0.09,0.34)$ \\
\hline Executive functioning composite & $0.39(0.32,0.47)$ & $0.42(0.35,0.50)$ & $0.39(0.30,0.49)$ & $0.40(0.31,0.49)$ & $0.13(0.05,0.22)$ & $0.15(0.06,0.25)$ \\
\hline Visuospatial composite & $0.15(0.07,0.24)$ & $0.17(0.09,0.29)$ & $0.07(0.01,0.14)$ & $0.06(0.01,0.14)$ & $0.08(0.00,0.18)$ & $0.10(0.02,0.22)$ \\
\hline MRI Hippocampal volume/ TIV ratio & $0.39(0.33,0.46)$ & $0.41(0.35,0.47)$ & $0.37(0.28,0.47)$ & $0.37(0.28,0.47)$ & $0.24(0.14,0.34)$ & $0.25(0.15,0.36)$ \\
\hline MRI AD signature region thickness & $0.39(0.33,0.45)$ & $0.42(0.36,0.48)$ & $0.28(0.19,0.39)$ & $0.30(0.20,0.43)$ & $0.27(0.16,0.39)$ & $0.29(0.19,0.41)$ \\
\hline
\end{tabular}

Data are presented as the median (95\% confidence interval [CI]) of R-squared values for the simple models for CSF p-tau181 and CSF p-tau217. Values were taken from the ridge regression models over 1000 bootstrapped samples. 
Supplementary figure 1. Concordance between tau PET in the entorhinal cortex, CSF ptau-217 and plasma p-tau217 in BioFINDER-2
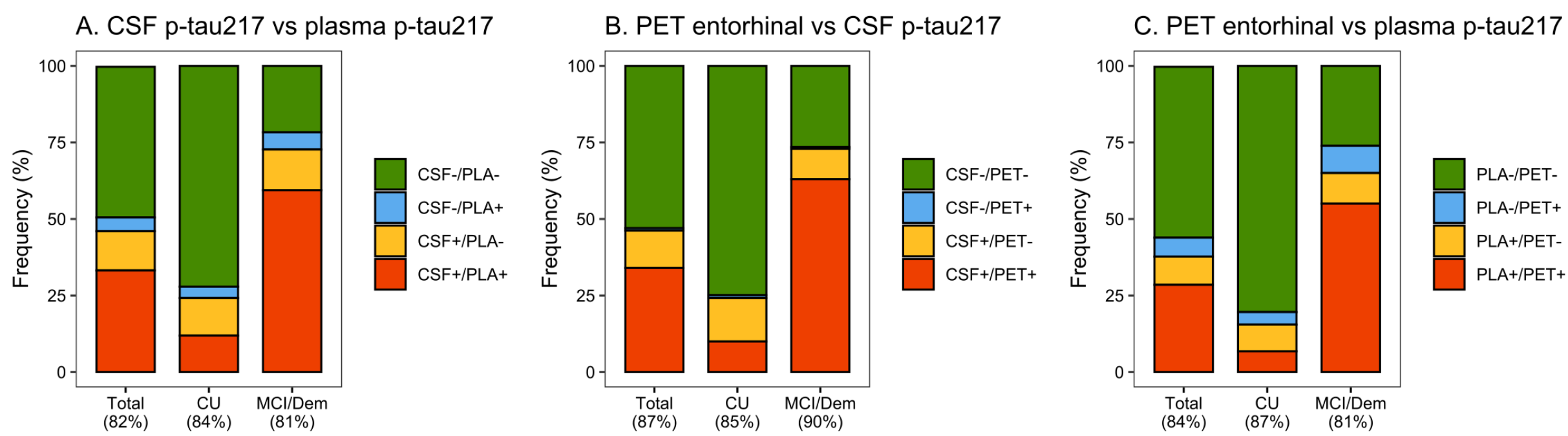

The graphs represent concordance rates between different tau biomarkers in BioFINDER-2: A) CSF p-tau217 vs plasma p-tau217, B) Tau PET in the entorhinal cortex vs CSF p-tau217, and C Tau PET in the entorhinal cortex vs plasma p-tau217. Cut-offs for both tau PET and fluid biomarkers are based on the mean $+(2 \mathrm{x}$ standard deviation) in $\mathrm{A} \beta$-negative cognitively normal individuals (see Methods section for further detail). 
Supplementary figure 2 Differential associations of entorhinal cortex tau PET vs CSF p-tau217 with Alzheimer-related features in BioFINDER-2
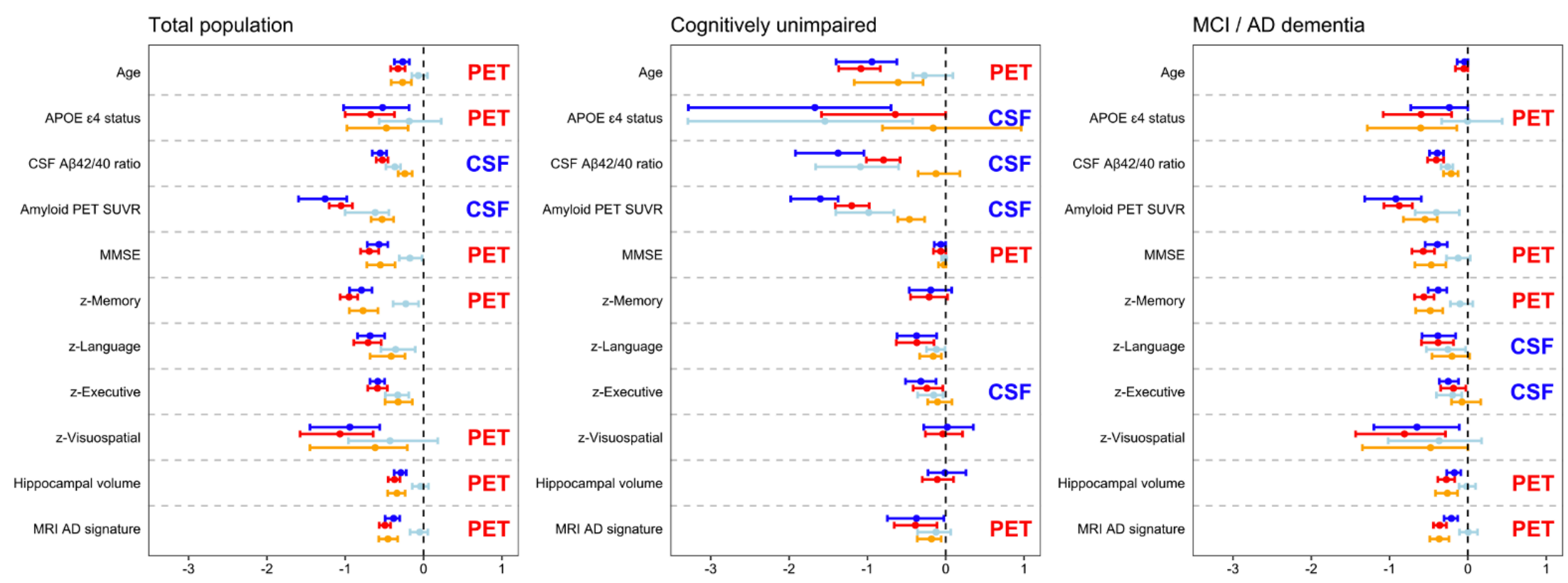

CSF $\beta \rightarrow$ PET $\beta \multimap \operatorname{CSF} \beta$ (combined model) $\rightarrow$ PET $\beta$ (combined model)

\section{Supplementary figure 2.}

Graphs display the differential association of entorhinal cortex tau PET vs CSF p-tau217 with AD-related features in BioFINDER-2. Median and 95\% confidence intervals (CI) of $\beta$-coefficients are plotted from the following ridge regression models: (i) AD-related feature predicted by CSF p-tau217 (dark blue, simple model), (ii) AD-related feature predicted by tau PET (dark red, simple model), (iii) AD-related feature predicted by the combination of CSF p-tau217 (light blue) and tau PET (orange, combined model). In case a feature was non-significant for both tau biomarkers in the simple models (i.e., $95 \%$ CIs crossed the 0 -line), no combined model was performed. All models were adjusted for age and sex, and cognitive tests were additionally adjusted for education. The $\beta$-coefficients for age, $A P O E \varepsilon 4$ carriership and amyloid PET global measures were multiplied by -1 for visualisation purposes.

Three criteria were used to determine whether one biomarker showed a stronger association with an AD feature compared to the other tau biomarker: (i) the $95 \% \mathrm{CI}$ between the simple models are non-overlapping (stronger association for the biomarker with more extreme values), (ii) the $95 \% \mathrm{CI}$ in the simple or combined models cross the 0-line (stronger association for the biomarker that did not cross the 0-line), and (iii) there is a lack of a significant drop in the $\beta$-coefficient from the combined model relative to the simple model for one of the two tau biomarkers, reflected by overlapping 95\% CIs between the simple and combined model for one tau biomarker in the presence of nonoverlapping 95\% CIs for the other tau biomarker (stronger association for the biomarker with overlapping 95\% CIs). If one or more of these criteria were met, this was marked on the right side of plots. 


\section{REFERENCES}

1. Scheltens P, De Strooper B, Kivipelto M, et al. Alzheimer's disease. Lancet [online ahead of print].

2. Busche MA, Hyman BT. Synergy between amyloid-beta and tau in Alzheimer's disease. Nat Neurosci. 2020.

3. Jack CR, Jr., Holtzman DM. Biomarker modeling of Alzheimer's disease. Neuron. 2013;80(6):1347-1358.

4. Mattsson-Carlgren N, Andersson E, Janelidze S, et al. Abeta deposition is associated with increases in soluble and phosphorylated tau that precede a positive Tau PET in Alzheimer's disease. Sci Adv. 2020;6(16):eaaz2387.

5. Nelson PT, Alafuzoff I, Bigio EH, et al. Correlation of Alzheimer disease neuropathologic changes with cognitive status: a review of the literature. $J$ Neuropathol Exp Neurol. 2012;71(5):362-381.

6. Spires-Jones TL, Hyman BT. The intersection of amyloid beta and tau at synapses in Alzheimer's disease. Neuron. 2014;82(4):756-771.

7. Savva GM, Wharton SB, Ince PG, et al. Age, neuropathology, and dementia. $N$ Engl J Med. 2009;360(22):2302-2309.

8. Ashton NJ, Leuzy A, Karikari TK, et al. The validation status of blood biomarkers of amyloid and phospho-tau assessed with the 5-phase development framework for AD biomarkers. Eur J Nucl Med Mol Imaging [online ahead of print].

9. Bischof GN, Dodich A, Boccardi M, et al. Clinical validity of second-generation tau PET tracers as biomarkers for Alzheimer's disease in the context of a structured 5phase development framework. Eur J Nucl Med Mol Imaging [online ahead of print].

10. Leuzy A, Ashton NJ, Mattsson-Carlgren N, et al. 2020 update on the clinical validity of cerebrospinal fluid amyloid, tau, and phospho-tau as biomarkers for Alzheimer's disease in the context of a structured 5-phase development framework. Eur J Nucl Med Mol Imaging [online ahead of print].

11. Wolters EE, Dodich A, Boccardi M, et al. Clinical validity of increased cortical uptake of [(18)F]flortaucipir on PET as a biomarker for Alzheimer's disease in the context of a structured 5-phase biomarker development framework. Eur J Nucl Med Mol Imaging [online ahead of print].

12. Janelidze S, Mattsson N, Palmqvist S, et al. Plasma P-tau181 in Alzheimer's disease: relationship to other biomarkers, differential diagnosis, neuropathology and longitudinal progression to Alzheimer's dementia. Nat Med. 2020;26(3):379-386.

13. Janelidze S, Stomrud E, Smith R, et al. Cerebrospinal fluid p-tau 217 performs better than p-tau181 as a biomarker of Alzheimer's disease. Nat Commun. 2020;11(1):1683.

14. Karikari TK, Pascoal TA, Ashton NJ, et al. Blood phosphorylated tau 181 as a biomarker for Alzheimer's disease: a diagnostic performance and prediction modelling study using data from four prospective cohorts. Lancet Neurol. 2020;19(5):422-433.

15. Mielke MM, Hagen CE, Xu J, et al. Plasma phospho-tau181 increases with Alzheimer's disease clinical severity and is associated with tau- and amyloid-positron emission tomography. Alzheimers Dement. 2018;14(8):989-997.

16. Palmqvist S, Janelidze S, Quiroz YT, et al. Discriminative Accuracy of Plasma Phospho-tau217 for Alzheimer Disease vs Other Neurodegenerative Disorders. JAMA. 2020;324(8):772-781. 
17. Barthelemy NR, Li Y, Joseph-Mathurin N, et al. A soluble phosphorylated tau signature links tau, amyloid and the evolution of stages of dominantly inherited Alzheimer's disease. Nat Med. 2020;26(3):398-407.

18. Thijssen EH, La Joie R, Wolf A, et al. Diagnostic value of plasma phosphorylated tau181 in Alzheimer's disease and frontotemporal lobar degeneration. Nat Med. 2020;26(3):387-397.

19. Hostetler ED, Walji AM, Zeng Z, et al. Preclinical Characterization of 18F-MK-6240, a Promising PET Tracer for In Vivo Quantification of Human Neurofibrillary Tangles. J Nucl Med. 2016;57(10):1599-1606.

20. Kuwabara H, Comley RA, Borroni E, et al. Evaluation of (18)F-RO-948 PET for Quantitative Assessment of Tau Accumulation in the Human Brain. J Nucl Med. 2018;59(12):1877-1884.

21. Lemoine L, Leuzy A, Chiotis K, Rodriguez-Vieitez E, Nordberg A. Tau positron emission tomography imaging in tauopathies: The added hurdle of off-target binding. Alzheimers Dement (Amst). 2018;10:232-236.

22. Marquie M, Normandin MD, Vanderburg CR, et al. Validating novel tau positron emission tomography tracer [F-18]-AV-1451 (T807) on postmortem brain tissue. Ann Neurol. 2015;78(5):787-800.

23. Xia CF, Arteaga J, Chen G, et al. [(18)F]T807, a novel tau positron emission tomography imaging agent for Alzheimer's disease. Alzheimers Dement. 2013;9(6):666-676.

24. Mattsson N, Scholl M, Strandberg O, et al. (18)F-AV-1451 and CSF T-tau and P-tau as biomarkers in Alzheimer's disease. EMBO Mol Med. 2017;9(9):1212-1223.

25. Meyer PF, Pichet Binette A, Gonneaud J, Breitner JCS, Villeneuve S. Characterization of Alzheimer Disease Biomarker Discrepancies Using Cerebrospinal Fluid Phosphorylated Tau and AV1451 Positron Emission Tomography. JAMA Neurol. 2020;77(4):508-516.

26. Reimand J, Collij L, Scheltens P, Bouwman F, Ossenkoppele R, Alzheimer's Disease Neuroimaging I. Association of amyloid-beta CSF/PET discordance and tau load 5 years later. Neurology. 2020;95(19):e2648-e2657.

27. Janelidze S, Berron D, Smith R, et al. Associations of Plasma Phospho-Tau217 Levels With Tau Positron Emission Tomography in Early Alzheimer Disease. JAMA Neurol. 2021;78(2):149-156.

28. Mattsson-Carlgren N, Janelidze S, Palmqvist S, et al. Longitudinal plasma p-tau217 is increased in early stages of Alzheimer's disease. Brain. 2020;143(11):3234-3241.

29. Ashton NJ, Pascoal TA, Karikari TK, et al. Plasma p-tau231: a new biomarker for incipient Alzheimer's disease pathology. Acta Neuropathol [online ahead of print].

30. Moscoso A, Grothe MJ, Ashton NJ, et al. Time course of phosphorylated-tau181 in blood across the Alzheimer's disease spectrum. Brain. 2021;144(1):325-339.

31. Suarez-Calvet M, Karikari TK, Ashton NJ, et al. Novel tau biomarkers phosphorylated at T181, T217 or T231 rise in the initial stages of the preclinical Alzheimer's continuum when only subtle changes in Abeta pathology are detected. EMBO Mol Med. 2020;12(12):e12921.

32. Jack CR, Jr., Bennett DA, Blennow K, et al. NIA-AA Research Framework: Toward a biological definition of Alzheimer's disease. Alzheimers Dement. 2018;14(4):535-562.

33. Jack CR, Jr., Bennett DA, Blennow K, et al. A/T/N: An unbiased descriptive classification scheme for Alzheimer disease biomarkers. Neurology. 2016;87(5):539547. 
34. Fleisher AS, Pontecorvo MJ, Devous MD, Sr., et al. Positron Emission Tomography Imaging With [18F]flortaucipir and Postmortem Assessment of Alzheimer Disease Neuropathologic Changes. JAMA Neurol. 2020.

35. de Wilde A, Reimand J, Teunissen CE, et al. Discordant amyloid-beta PET and CSF biomarkers and its clinical consequences. Alzheimers Res Ther. 2019;11(1):78.

36. Fagan AM, Mintun MA, Mach RH, et al. Inverse relation between in vivo amyloid imaging load and cerebrospinal fluid Abeta42 in humans. Ann Neurol. 2006;59(3):512-519.

37. Palmqvist S, Mattsson N, Hansson O, Alzheimer's Disease Neuroimaging I. Cerebrospinal fluid analysis detects cerebral amyloid-beta accumulation earlier than positron emission tomography. Brain. 2016;139(Pt 4):1226-1236.

38. Landau SM, Lu M, Joshi AD, et al. Comparing positron emission tomography imaging and cerebrospinal fluid measurements of beta-amyloid. Ann Neurol. 2013;74(6):826-836.

39. Palmqvist S, Scholl M, Strandberg O, et al. Earliest accumulation of beta-amyloid occurs within the default-mode network and concurrently affects brain connectivity. Nat Commun. 2017;8(1):1214.

40. Sala A, Nordberg A, Rodriguez-Vieitez E, Alzheimer's Disease Neuroimaging I. Longitudinal pathways of cerebrospinal fluid and positron emission tomography biomarkers of amyloid-beta positivity. Mol Psychiatry. 2020.

41. Reimand J, Boon BDC, Collij LE, et al. Amyloid-beta PET and CSF in an autopsyconfirmed cohort. Ann Clin Transl Neurol. 2020;7(11):2150-2160.

42. Krut JJ, Zetterberg H, Blennow K, et al. Cerebrospinal fluid Alzheimer's biomarker profiles in CNS infections. J Neurol. 2013;260(2):620-626.

43. Mori F, Rossi S, Sancesario G, et al. Cognitive and cortical plasticity deficits correlate with altered amyloid-beta CSF levels in multiple sclerosis.

Neuropsychopharmacology. 2011;36(3):559-568.

44. Leuzy A, Smith R, Ossenkoppele R, et al. Diagnostic Performance of RO948 F 18 Tau Positron Emission Tomography in the Differentiation of Alzheimer Disease From Other Neurodegenerative Disorders. JAMA Neurol. 2020.

45. Ossenkoppele R, Rabinovici GD, Smith R, et al. Discriminative Accuracy of [18F]flortaucipir Positron Emission Tomography for Alzheimer Disease vs Other Neurodegenerative Disorders. JAMA. 2018;320(11):1151-1162.

46. Pascoal TA, Therriault J, Benedet AL, et al. 18F-MK-6240 PET for early and late detection of neurofibrillary tangles. Brain. 2020.

47. Jack CR, Jr., Wiste HJ, Schwarz CG, et al. Longitudinal tau PET in ageing and Alzheimer's disease. Brain. 2018;141(5):1517-1528.

48. Pontecorvo MJ, Devous MD, Kennedy I, et al. A multicentre longitudinal study of flortaucipir $(18 \mathrm{~F})$ in normal ageing, mild cognitive impairment and Alzheimer's disease dementia. Brain. 2019;142(6):1723-1735.

49. Smith R, Strandberg O, Mattsson-Carlgren N, et al. The accumulation rate of tau aggregates is higher in females and younger amyloid-positive subjects. Brain. 2020;143(12):3805-3815.

50. Mattsson-Carlgren N, Leuzy A, Janelidze S, et al. The implications of different approaches to define AT(N) in Alzheimer disease. Neurology. 2020;94(21):e2233e2244.

51. Lowe VJ, Lundt ES, Albertson SM, et al. Tau-positron emission tomography correlates with neuropathology findings. Alzheimers Dement. 2020;16(3):561-571.

52. Jin M, Shepardson N, Yang T, Chen G, Walsh D, Selkoe DJ. Soluble amyloid betaprotein dimers isolated from Alzheimer cortex directly induce Tau 
hyperphosphorylation and neuritic degeneration. Proc Natl Acad Sci U S A. 2011;108(14):5819-5824.

53. Spillantini MG, Crowther RA, Goedert M. Comparison of the neurofibrillary pathology in Alzheimer's disease and familial presenile dementia with tangles. Acta Neuropathol. 1996;92(1):42-48.

54. Salloway S, Sperling R, Fox NC, et al. Two phase 3 trials of bapineuzumab in mildto-moderate Alzheimer's disease. N Engl J Med. 2014;370(4):322-333.

55. Sevigny $\mathrm{J}$, Chiao $\mathrm{P}$, Bussiere $\mathrm{T}$, et al. Addendum: The antibody aducanumab reduces Abeta plaques in Alzheimer's disease. Nature. 2017;546(7659):564.

56. Ostrowitzki S, Lasser RA, Dorflinger E, et al. A phase III randomized trial of gantenerumab in prodromal Alzheimer's disease. Alzheimers Res Ther. 2017;9(1):95.

57. Sperling RA, Donohue MC, Raman R, et al. Association of Factors With Elevated Amyloid Burden in Clinically Normal Older Individuals. JAMA Neurol. 2020;77(6):735-745.

58. Mintun MA, Lo AC, Duggan Evans C, et al. Donanemab in Early Alzheimer's Disease. N Engl J Med. 2021.

59. McKhann GM, Knopman DS, Chertkow H, et al. The diagnosis of dementia due to Alzheimer's disease: recommendations from the National Institute on AgingAlzheimer's Association workgroups on diagnostic guidelines for Alzheimer's disease. Alzheimers Dement. 2011;7(3):263-269.

60. Albert MS, DeKosky ST, Dickson D, et al. The diagnosis of mild cognitive impairment due to Alzheimer's disease: recommendations from the National Institute on Aging-Alzheimer's Association workgroups on diagnostic guidelines for Alzheimer's disease. Alzheimers Dement. 2011;7(3):270-279.

61. Choi SE, Mukherjee S, Gibbons LE, et al. Development and validation of language and visuospatial composite scores in ADNI. Alzheimers Dement (N Y).

2020;6(1):e12072.

62. Crane PK, Trittschuh E, Mukherjee S, et al. Incidence of cognitively defined lateonset Alzheimer's dementia subgroups from a prospective cohort study. Alzheimers Dement. 2017;13(12):1307-1316.

63. Ossenkoppele R, Smith R, Ohlsson T, et al. Associations between tau, Abeta, and cortical thickness with cognition in Alzheimer disease. Neurology. 2019;92(6):e601e612.

64. Weiner MW, Veitch DP. Introduction to special issue: Overview of Alzheimer's Disease Neuroimaging Initiative. Alzheimers Dement. 2015;11(7):730-733.

65. Ossenkoppele R, Leuzy A, Cho H, et al. The impact of demographic, clinical, genetic, and imaging variables on tau PET status. Eur J Nucl Med Mol Imaging. 2020.

66. Ossenkoppele R, Lyoo CH, Jester-Broms J, et al. Assessment of Demographic, Genetic, and Imaging Variables Associated With Brain Resilience and Cognitive Resilience to Pathological Tau in Patients With Alzheimer Disease. JAMA Neurol. 2020;77(5):632-642.

67. Sled JG ZA, Evans AC. A nonparametric method for automatic correction of intensity nonuniformity in MRI data. IEEE Trans Med Imaging. 1998;17:10.

68. Segonne F, Dale AM, Busa E, et al. A hybrid approach to the skull stripping problem in MRI. Neuroimage. 2004;22(3):1060-1075.

69. Fischl B, Salat DH, Busa E, et al. Whole brain segmentation: automated labeling of neuroanatomical structures in the human brain. Neuron. 2002;33(3):341-355.

70. Fischl B, Dale AM. Measuring the thickness of the human cerebral cortex from magnetic resonance images. Proc Natl Acad Sci U S A. 2000;97(20):11050-11055. 
71. Jack CR, Jr., Wiste HJ, Weigand SD, et al. Defining imaging biomarker cut points for brain aging and Alzheimer's disease. Alzheimers Dement. 2017;13(3):205-216.

72. Klunk WE, Koeppe RA, Price JC, et al. The Centiloid Project: standardizing quantitative amyloid plaque estimation by PET. Alzheimers Dement. 2015;11(1):1-15 e11-14.

73. Maass A, Landau S, Baker SL, et al. Comparison of multiple tau-PET measures as biomarkers in aging and Alzheimer's Disease. Neuroimage. 2017.

74. Jack CR, Wiste HJ, Botha H, et al. The bivariate distribution of amyloid-beta and tau: relationship with established neurocognitive clinical syndromes. Brain. 2019;142(10):3230-3242.

75. Cho H, Choi JY, Hwang MS, et al. In vivo cortical spreading pattern of tau and amyloid in the Alzheimer disease spectrum. Ann Neurol. 2016;80(2):247-258.

76. Johnson KA, Schultz A, Betensky RA, et al. Tau positron emission tomographic imaging in aging and early Alzheimer disease. Ann Neurol. 2016;79(1):110-119.

77. Scholl M, Lockhart SN, Schonhaut DR, et al. PET Imaging of Tau Deposition in the Aging Human Brain. Neuron. 2016;89(5):971-982.

78. Braak H, Braak E. Neuropathological stageing of Alzheimer-related changes. Acta Neuropathol. 1991;82(4):239-259.

79. Blennow K, Hampel H, Weiner M, Zetterberg H. Cerebrospinal fluid and plasma biomarkers in Alzheimer disease. Nat Rev Neurol. 2010;6(3):131-144.

80. Lifke V, Kollmorgen G, Manuilova E, et al. Elecsys((R)) Total-Tau and Phospho-Tau (181P) CSF assays: Analytical performance of the novel, fully automated immunoassays for quantification of tau proteins in human cerebrospinal fluid. Clin Biochem. 2019;72:30-38.

81. Mattsson N, Insel PS, Donohue M, et al. Independent information from cerebrospinal fluid amyloid-beta and florbetapir imaging in Alzheimer's disease. Brain. 2015;138(Pt 3):772-783.

82. Friedman J, Hastie T, Tibshirani R. The elements of statistical learning. New York: Springer Series in Statistics; 2009.

83. Brieman JF, J.; Stone, C.J.; Olshen, R.A. Classification and Regression Trees. Taylor \& Francis; 1984. 